\title{
System FR: Formalized Foundations for the Stainless Verifier
}

\author{
JAD HAMZA, EPFL, Switzerland \\ NICOLAS VOIROL, EPFL, Switzerland \\ VIKTOR KUNČAK, EPFL, Switzerland
}

\begin{abstract}
We present the design, implementation, and foundation of a verifier for higher-order functional programs with generics and recursive data types. Our system supports proving safety and termination using preconditions, postconditions and assertions. It supports writing proof hints using assertions and recursive calls. To formalize the soundness of the system we introduce System FR, a calculus supporting System F polymorphism, dependent refinement types, and recursive types (including recursion through contravariant positions of function types). Through the use of sized types, System FR supports reasoning about termination of lazy data structures such as streams. We formalize a reducibility argument using the Coq proof assistant and prove the soundness of a type-checker with respect to call-by-value semantics, ensuring type safety and normalization for typeable programs. Our program verifier is implemented as an alternative verification-condition generator for the Stainless tool, which relies on the Inox SMT-based solver backend for automation. We demonstrate the efficiency of our approach by verifying a collection of higher-order functional programs comprising around 14000 lines of polymorphic higher-order Scala code, including graph search algorithms, basic number theory, monad laws, functional data structures, and assignments from popular Functional Programming MOOCs.
\end{abstract}

CCS Concepts: • Theory of computation $\rightarrow$ Logic and verification; Programming logic; Type theory; - Software and its engineering $\rightarrow$ Software verification; • Computing methodologies $\rightarrow$ Theorem proving algorithms.

Additional Key Words and Phrases: software verification, dependent types, recursive types, System F, SMT

ACM Reference Format:

Jad Hamza, Nicolas Voirol, and Viktor Kunčak. 2019. System FR: Formalized Foundations for the Stainless Verifier. Proc. ACM Program. Lang. 3, OOPSLA, Article 166 (October 2019), 30 pages. https://doi.org/10.1145/ 3360592

\section{INTRODUCTION}

Automatically verifying the correctness of higher-order programs is a long-standing problem that arises in most programming languages and proof assistants. Despite extensive research in program verifiers and proof assistants [Abel 2010; Bertot and Castéran 2004a; Brady 2013; Gordon and Melham 1993; Harrison 2009, 2017; Leino 2010; Nipkow et al. 2002a; Norell 2007; Swamy et al. 2013; Vazou et al. 2014] there remain significant challenges and trade-offs in checking safety and termination. A motivation for our work are implementations that verify polymorphic functional programs using SMT solvers [Suter et al. 2011; Vazou et al. 2014]. To focus on foundations, we look at simpler verifiers that do not perform invariant inference and are mostly based on unfolding recursive definitions and encoding of higher-order functions into SMT theories [Blanc and Kuncak 2015; Suter et al. 2011; Voirol et al. 2015]. A recent implementation of such a verifier is the Stainless

Authors' addresses: Jad Hamza, LARA, EPFL, Switzerland, jad.hamza@epfl.ch; Nicolas Voirol, LARA, EPFL, Switzerland, nicolas.voirol@epfl.ch; Viktor Kunčak, EPFL IC LARA - INR 318 - Station 14, EPFL, Switzerland, viktor.kuncak@epfl.ch.

This work is licensed under a Creative Commons Attribution-ShareAlike 4.0 International License.

(c) 2019 Copyright held by the owner/author(s).

2475-1421/2019/10-ART166

https://doi.org/10.1145/3360592

Proc. ACM Program. Lang., Vol. 3, No. OOPSLA, Article 166. Publication date: October 2019. 
system [LARA 2019], which handles a subset of Scala [Odersky et al. 2008]. The goal of Stainless is to verify that function contracts hold and that all functions terminate. It was shown [Hupel and Kuncak 2016] how to map certain patterns of specified Scala programs into Isabelle/HOL. Whereas this approach ensures soundness, it does not reuse the reasoning of Stainless and it can verify only some of the programs that Stainless verifier can prove. The present paper seeks to provide direct foundations for verification and termination checking of functional programs with a rich set of features for purely functional programming including non-monotonic data types. On the other hand, our calculus does not aspire to directly support effects for which there exist excellent other systems [Swamy et al. 2013].

The subtleties of ensuring function termination have been an initial impetus for the calculus we present. Termination is desirable for many executable functions in programs and is even more important in formal specifications. A non-terminating function definition such as $f(x)=1+f(x)$ could be easily mapped to a contradiction and violate the conservative extension principle for definitions. Yet termination in the presence of higher-order functions and data types is challenging to ensure. For example, when using non-monotonic recursive types, terms can diverge even without the explicit use of recursive functions, as illustrated by the following snippet of Scala code:

case class $\mathrm{D}(\mathrm{f}: \mathrm{D} \Rightarrow$ Unit $) / /$ non-monotonic recursive type
def $\mathrm{g}(\mathrm{d}: \mathrm{D}):$ Unit $=\mathrm{d} . \mathrm{f}(\mathrm{d}) / /$ non-recursive function definition
$\mathrm{g}(\mathrm{D}(\mathrm{g})) / /$ diverging term, reduces to $D(\mathrm{~g}) . f(D(\mathrm{~g}))$ and then again to $\mathrm{g}(D(\mathrm{~g}))$

Furthermore, even though the concept of termination for all function inputs is an intuitively clear property, its modular definition is subtle: a higher order function $g$ taking another function $f$ as an argument should terminate when given any terminating function $f$, which, in turn, can be applied to expressions involving further calls to $g$. The quest for solid foundations for termination led us to type theoretic techniques, where reducibility method has long been used to show strong normalization of expressive calculi [Tait 1967], [Girard 1990, Chapter 6], [Harper 2016]. As a natural framework for analyzing support for first-class functions with preconditions and post-conditions we embraced the ideas of refinement dependent types similar to those in Liquid Haskell [Vazou et al 2014] with refinement-based notion of subtyping. To explain proof obligation generation in the higher-order case (including the question of which assumptions should be visible when checking a given assertion), we resorted to well-known dependent (П) function types. To support parametric polymorphism we incorporated type quantifiers, as in System F [Girard 1971, 1990]. We found that the presence of refinement types allowed us to explain soundness of well-founded recursion based on user-defined measures. The recursion in programs is thus not syntactically restricted as in, e.g., System F. To provide expressive support for iterative unfolding of recursive functions, we introduced rules to make function bodies available while type checking of recursive functions. For recursive type definitions, many existing systems introduce separate notions of inductive and co-inductive definitions. We found this distinction less natural for developers and chose to support expressive recursive types (without a necessary restriction to positive recursion) using sized types [Abel 2010]. We draw inspiration from a number of existing systems, yet our solution is a new sound combination of features that work nicely together.

We combined these features into a new type system, System FR, which we present as a bidirectional type checking algorithm. The algorithm generates type checking and type inference goals by traversing terms and types, until it reaches a point where it has to check that a given term evaluates to true. This typically arises when we want to check that a term $t$ has a refinement type $\{x: T \mid b\}$, which is the case when $t$ has type $T$, and when the term $b$ evaluates to true in the context where $x$ equals $t$. Following the tradition of SMT-based verifiers [Barnett et al. 2004; Detlefs et al. 1998], we use the term verification condition (VC) to refer to a term that should evaluate to true. 
We prove the soundness of our type system using a reducibility interpretation of types. The goal of our verification system is to ensure that a given term belongs to the semantic denotation of a given type. For simple types such as natural numbers, this denotation is the set of untyped lambda calculus terms that evaluate, in a finite number of steps, to a non-negative integer. For function types the denotation are, as is typical in reducibility approaches, terms that, when applied to terms in denotation of argument type, evaluate to terms in the denotation of the result type. Such denotation gives us a unified framework for function contracts expressed as refinement types. The approach ensures termination of programs because the semantics of types only contain terms that are terminating in call-by-value semantics.

We have formally proven using the Coq proof assistant [Bertot and Castéran 2004a] the soundness of our typing algorithm, implying that when verification conditions generated for checking that a term $t$ belongs to a type $T$ are semantically valid, the term $t$ belongs to the semantic denotation of the type $T$. The bidirectional typing algorithm handles the expressive types in a deterministic and predictable way, which enables good and localized error reporting to the user. To solve generated verification conditions, we use existing implementation invoking the Inox solver ${ }^{1}$ that reduces higher-order queries to the first-order language of SMT solvers [Voirol et al. 2015]. Our semantics of types provides a definition of soundness for such solvers; any solver that respects the semantics can be used with our verification condition generator. Our bidirectional type checking algorithm thus becomes a new, trustworthy verification condition generator for Stainless. We were successful in verifying many existing Stainless benchmarks using the new approach.

We summarize our contributions as follows:

- We present a rich type system, called System FR, that combines System F with dependent types, refinements, equality types, and recursive types (Sections 3 and 4).

- We define a bidirectional type-checking algorithm for System FR (Section 5). Our algorithm generates verification conditions that are solved by the (existing) SMT-based solver Inox.

- We prove ${ }^{2}$ soundness of our bidirectional type-checking algorithm that reduces program correctness to proving that certain formulas always evaluate to true (Section 6). Our formalization also supports additional expressive notions, such as infinite intersections and unions as well as refinement conditions given by non-emptiness of an arbitrary type.

- We built a verification condition generator based on these foundations and evaluated it on around 14k lines of benchmarks (Section 7), showing that generating proof obligations using type checking is effective in practice.

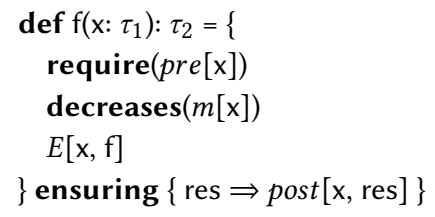

Fig. 1. Template of a recursive function with user-given contracts and a decreasing measure.

\section{EXAMPLES OF PROGRAM VERIFICATION AND TERMINATION CHECKING}

Our goal is to verify correctness and termination of pure Scala functions written as in Figure 1. $\operatorname{pre}[\mathrm{x}]$ is the precondition of the function $\mathrm{f}$, and is written by the user in the same language as

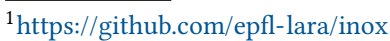

${ }^{2}$ https://github.com/epfl-lara/SystemFR/tree/oopsla2019
} 
the body of $f$. The precondition may contain arbitrary expressions and calls to other functions. Similarly, the user specifies in post the property that the results of the function should satisfy. To ensure termination of $f$ (which might call itself recursively), the user may also provide a measure using the decreases keyword, which is also an expression (of type Nat, the type of natural numbers) written in the same language. $\tau_{1}$ and $\tau_{2}$ may be arbitrary types, including function types or algebraic data types. Informally, the function is terminating and correct, if, for every value $v$ of type $\tau_{1}$ such that pre $[\mathrm{v}]$ evaluates to true, $f(v)$ returns (in a finite number of steps) a value res of type $\tau_{2}$ such that post $[\mathrm{v}, \mathrm{res}]$ evaluates to true. By using dependent and refinement types, this can be summarized by saying that the function $f$ has type: $\Pi x:\left\{x: \tau_{1} \mid \operatorname{pre}[x]\right\} .\left\{\right.$ res : $\tau_{2} \mid \operatorname{post}[x$, res $\left.]\right\}$.

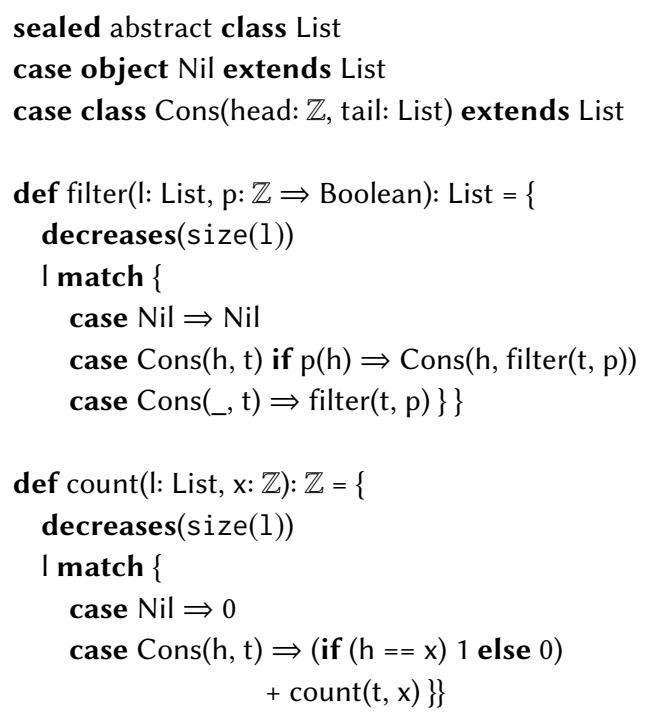

Fig. 2. The function filter filters elements of a list based on a predicate $p$, and count counts the number of occurrences of $x$ in a list.

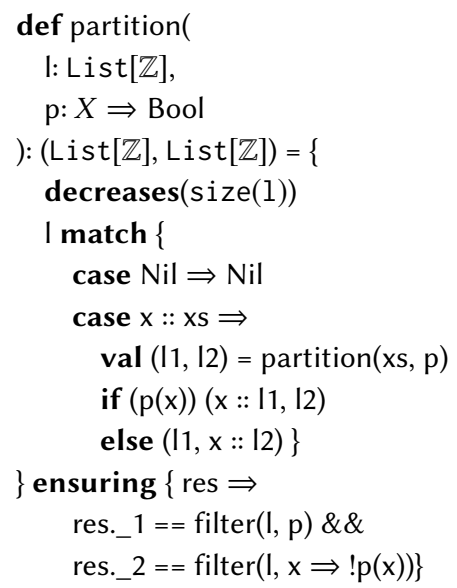

Fig. 3. A partition function specified using filter and with termination measure is given with size.

As an example, consider the list type as defined in Figure 2. We use $\mathbb{Z}$ to denote the type of integers (corresponding to Scala's BigInt in actual source code). The function filter filters elements from a list, while count counts the number of occurrences of an integer in the list. These two functions have no pre- or postconditions. The decreases clauses specify that the functions terminate because the size of the list decreases at each recursive call.

Using these functions we define partition in Figure 3, which takes a list 1 of integers and partitions it according to a predicate $\mathrm{p}: \mathbb{Z} \Rightarrow$ Bool. We prove in the postcondition that partitioning coincides with applying filter to the list with $p$ and its negation.

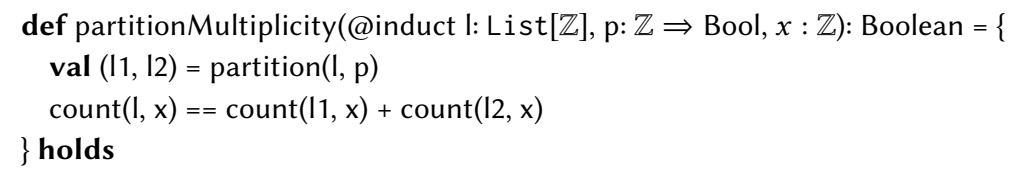

Fig. 4. A proof (by induction on 1) that partitioning a list preserves the multiplicity of each element. 

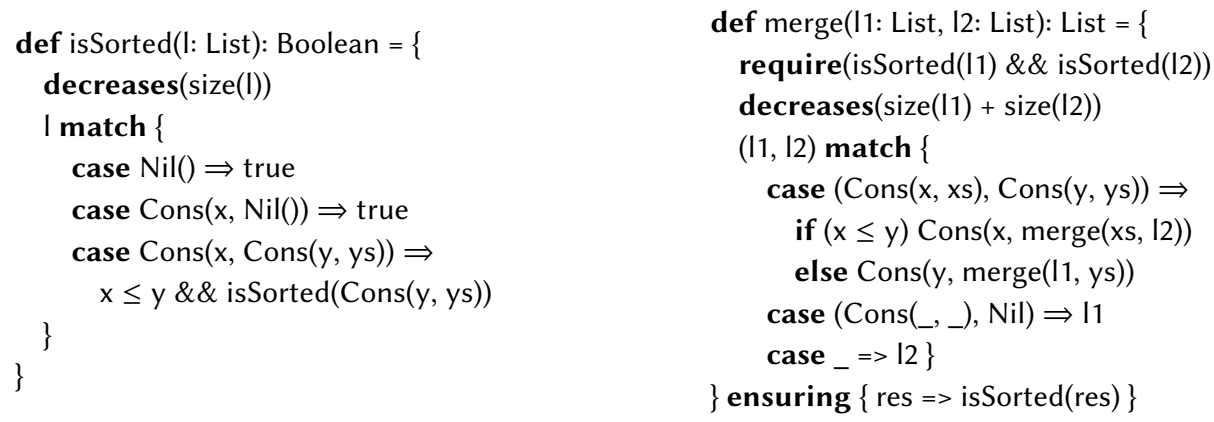

Fig. 5. A function that checks whether a list is sorted and a function that merges two sorted lists

Figure 4 shows a theorem that partition also preserves the multiplicity of each element. We use here count to state the property, but we could have used multisets instead (a type which is natively supported in Stainless). The holds keyword is a shorthand for ensuring \{ res $\Rightarrow$ res \}. The @induct annotation instructs the system to add a recursive call to partitionMultiplicity on the tail of 1 when 1 is not empty. This gives us access to the multiplicity property for the tail of 1 , which the system can then use automatically to prove that the property holds for 1 itself. This corresponds to a proof by induction on 1 .

Figure 5 shows a function isSorted that checks whether a list is sorted, and a function merge that combines two sorted lists in a sorted list. When given the above input, the system proves the termination of all functions, establishes that postconditions of functions hold, and shows that the theorem holds, without any user interaction or additional annotations. For the merge function, the postcondition might seem too weak to establish that e.g. Cons ( $x$, merge (xs, 12)) is sorted just based on the fact that merge ( $x s, 12)$ is sorted. However, since we put the definition of merge while type-checking the body of merge in the context, it is possible to establish that $x$ is smaller than the head of merge (xs, 12). We give more details on this feature of our system, called body-visible recursion, in Section 5.3.

\subsection{Reasoning about Streams}

Our system also supports reasoning about infinite data structures, including streams that are computed on demand. These data structures are challenging to deal with because even defining termination of an infinite stream is non-obvious, especially in absence of a concrete operation that uses the stream. Given some type X, Stream $[X]$ represents the type of infinite streams containing elements in X. In a mainstream call-by-value language such as Scala, this type can be defined as:

case class Stream $[X]$ (head: $X$, tail: ()$\Rightarrow$ Stream $[X])$

For the sake of concise syntax, we typeset a function taking unit, (u:Unit)=>e, using Scala's syntax ()$\Rightarrow e$ for a function of zero parameters. Given a stream s: Stream $[X]$, we can call s. head to get the head of the stream (which is of type X), or s. tail to get the tail of the stream (which is of type ()$\Rightarrow$ Stream $[X])$. We can use recursion to define streams, as shown in figures $6,8,7$. The @erasable annotation is used to mark the erasable parameters $n$ of these functions. These parameters are used as annotations to guide our type-checker, but they do not influence the computation and can be erased at runtime. For instance, an erased version of constant (without erasable code and without type annotation) looks like:

def $\operatorname{constant}(x)=\operatorname{Stream}(x,() \Rightarrow \operatorname{constant}(x))$ 


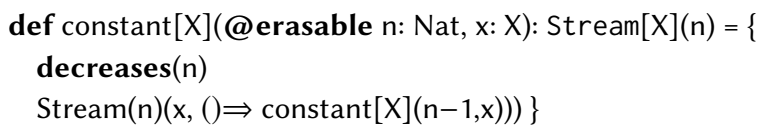

Fig. 6. Constant stream

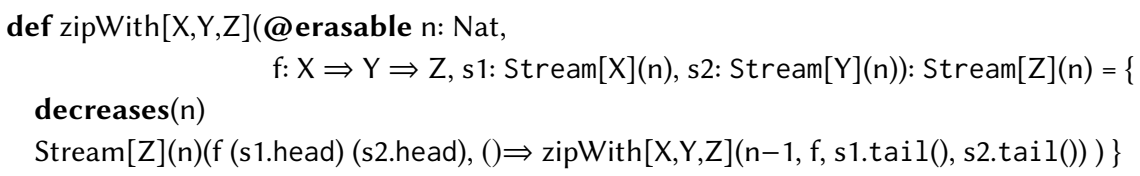

Fig. 7. Zip function that combines elements of two streams using a two-argument function $f$

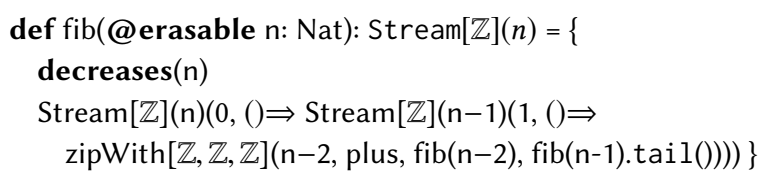

Fig. 8. Fibonacci stream defined using zipWith

Informally, we can say that the constant stream is terminating. Indeed, it has the interesting property that, despite the recursion, for every $n \in \mathbb{N}$, we can take the first $n$ elements in finite time (no divergence in the computation). We say that constant $(x)$ is an $n$-non-diverging stream. Moreover, when a stream is $n$-non-diverging for every $n \in \mathbb{N}$, we simply say that it is non-diverging, which means that we can take as many elements as we want without diverging, which is the case for constant $(x)$. Note that non-divergence of constant cannot be shown by defining a measure on its argument $x$ that strictly decreases on each recursive call, because constant is called recursively on the exact same argument $x$. Instead, we define a measure on the erasable argument $n$ of the annotated version. This corresponds to using type-based termination [Abel 2007, 2008; Barthe et al. 2008], where the type of the function for the recursive call is smaller than the type of the caller. We expand on that technique in Section 5.2 .

In the annotated version of constant from Figure 6, the notation Stream[X](n) stands for streams of elements in $X$ which are $n$-non-diverging. The type of constant then states that constant can be called with any (erasable) parameter $n$ to build an $n$-non-diverging stream. Since parameter $n$ is computationally irrelevant, this proves that the erased version of constant returns a non-diverging stream.

We now give a variant of constant which is diverging:

def $\operatorname{badConstant}(\mathrm{x})=\operatorname{Stream}(\mathrm{x},() \Rightarrow \operatorname{badConstant}(\mathrm{x}) \cdot \operatorname{tail}())$

Indeed, given some $x$, a call to badConstant( $x)$.tail() ends up evaluating badConstant $(x) \cdot \operatorname{tail}()$ again, and would diverge.

The zipWith function in Figure 7 takes two streams and a function $f$. It creates a new stream by applying $f$ to pairs of elements taken from each stream. For zipWith, we can verify that as long as $f$ terminates on every input, and s1 and s2 are non-diverging streams, then zipWith returns a non-diverging stream. We can then use zipWith to define the well-known Fibonacci stream (Figure 8), an infinite stream containing the Fibonacci sequence: 0, 1, 1, 2, 3, 5, 8, 13, etc. We make use of a function plus: $\mathbb{Z} \Rightarrow \mathbb{Z} \Rightarrow \mathbb{Z}$ that computes the sum of two integers. Just like the constant 
streams, fib is a non-diverging stream. For instance, calling fib.tail().tail().tail(). head returns (in finite time) the number 2 from the Fibonacci sequence.

The important property that the type signature of zipWith ensures is that, for every $n \in$ Nat, if $s 1$ and s2 are $n$-non-diverging streams, then zipWith $f s 1$ s2 is $n$-non-diverging as well. Our type system can check this property and then use it to make sure that the definition of fib type-checks. We can also prove further properties of interest, e.g., that zipping two streams s1 and s2 with the function $(x: N a t) \Rightarrow(y: N a t) \Rightarrow x$ returns a stream that behaves as the stream $s 1$.

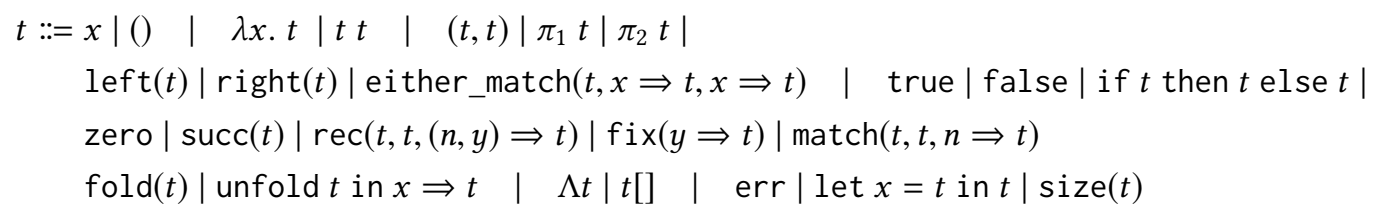

Fig. 9. Grammar for untyped lambda calculus terms

\section{SYNTAX AND OPERATIONAL SEMANTICS}

We now give a formal syntax for terms and show call-by-value operational semantics. This untyped lambda calculus with pairs, tagged unions, integers, booleans, and error values models programs that our verification system supports. It is Turing complete and rather conventional.

\subsection{Terms of an Untyped Calculus}

Let $\mathcal{V}$ be a set of variables. We let Terms be the set of all (untyped) terms (see Figure 9) which includes the unit term (), pairs, booleans, natural numbers, a recursor rec for iterating over natural numbers, a pattern matching operator match for natural numbers, a recursion operator $f i x$, an error term err to represent crashes. The recursor rec can be simulated using fix and match but we keep it in the paper for presenting examples.

The terms fold $(t)$ and unfold $t_{1}$ in $x \Rightarrow t_{2}$ are used to represent data structures (such as lists or streams), where 'fold()' plays the role of a constructor, and 'unfold in ' the role of a deconstructor. The terms $\Lambda t$ and $t[]$ are used to represent the erasure of type abstractions and type instantiation terms (for polymorphism) of the form $\Lambda \alpha . t$ and $t[\tau]$, where $\alpha$ is a type variable and $\tau$ is a type. These annotated terms will be introduced in a further section.

The term size $(t)$ is a special term to internalize the sizes of syntax trees of values (ignoring lambdas) of our language. It is used for measure of recursive functions such as the map examples on lists shown in Section 2.

Given a term $t$ we denote $\mathrm{fv}(t)$ the set of all free variables of $t$. Terms are considered up to renaming of locally bound variables (alpha-renaming).

\subsection{Call-by-Value Operational Semantics}

The set Val of values of our language is defined (inductively) to be zero, (), true, false, every variable $x$, every lambda term $\lambda x . t$ or $\Lambda t$, the terms of the form $\operatorname{succ}(v)$ or fold $(v)$ where $v \in \operatorname{Val}$, and the terms of the form $\left(v_{1}, v_{2}\right)$ where $v_{1}, v_{2} \in$ Val.

The call-by-value small-step relation between two terms $t_{1}, t_{2} \in$ Terms, written $t_{1} \hookrightarrow t_{2}$, is standard for the most part and given in Figure 10. Given a term $t$ and a value $v, t[x \mapsto v]$ denotes the term $t$ where every free occurrence of $x$ has been replaced by $v$. 


$$
\begin{aligned}
\tau::= & \text { Unit | Bool | Nat }|\top \quad| \quad \Pi x: \tau . \tau|\Sigma x: \tau . \tau| \tau+\tau \quad|\quad \forall x: \tau . \tau| \forall \alpha: \text { Type. } \tau \\
& \operatorname{Rec}(n)(\alpha \Rightarrow \tau) \quad|\quad\{x: \tau \mid t\}| t \equiv t
\end{aligned}
$$

Fig. 12. Grammar for types $\tau$, where $x \in \mathcal{V}$ is a term variable, $\alpha \in \mathcal{V}$ is a type variable ( $t$ denotes annotated terms of Figure 16 that complete the mutually recursive definition)

\section{TYPES, SEMANTICS AND REDUCIBILITY}

We give in Figure 12 the grammar for the types $\tau$ that our verification system supports. Given two types $\tau_{1}$ and $\tau_{2}$, we use the notation $\tau_{1} \rightarrow \tau_{2}$ for $\Pi x: \tau_{1} . \tau_{2}$ when $x$ is not a free variable of $\tau_{2}$. Similarly, we use the notation $\tau_{1} \times \tau_{2}$ for $\Sigma x: \tau_{1} . \tau_{2}$ when $x$ is not a free variable of $\tau_{2}$.

For recursive types, we introduce the notation:

$$
\operatorname{Rec}(\alpha \Rightarrow \tau) \triangleq \forall n: \text { Nat. } \operatorname{Rec}(n)(\alpha \Rightarrow \tau)
$$

Then, the type of (non-diverging) streams informally introduced in Section 2 can be understood as a notation, when $\mathrm{X}$ is a type, for: Stream $[\mathrm{X}] \triangleq \operatorname{Rec}(\alpha \Rightarrow \mathrm{X} \times($ Unit $\rightarrow \alpha))$. Similarly, for a natural number $n$, the type of $n$-non-diverging streams $\operatorname{Stream}_{n}[\mathrm{X}]$ is a notation for $\operatorname{Rec}(n)(\alpha \Rightarrow$ $\mathrm{X} \times($ Unit $\rightarrow \alpha))$. Using this notation, we can also define finite data structures such as lists of elements from $\mathrm{X}$, as follows: $\operatorname{List}[\mathrm{X}] \triangleq \operatorname{Rec}(\alpha \Rightarrow$ Unit $+\mathrm{X} \times \alpha)$.

We show in Section 4.3 that these types indeed correspond to streams and lists respectively.

Let Type be the set of all types. We define a (unary) logical relation on types to describe terms that do not get stuck (e.g. due to the error term err, or due to an ill-formed application such as 'true zero') and that terminate to a value of the given type. Our definition is inspired by the notion of reducibility or hereditary termination (see e.g. [Girard 1990; Harper 2016; Tait 1967]), which we use as a guiding principle for designing the type system and its extensions.

\subsection{Reduciblity for Closed Terms}

For each type $\tau$, we define in Figure 13 mutually recursively the sets of reducible values $\llbracket \tau \rrbracket_{\mathrm{v}}^{\theta}$ and reducible terms $\llbracket \tau \rrbracket_{\mathrm{t}}^{\theta}$. In that sense, a type $\tau$ can be understood as a specification that some terms satisfy (and some do not).

These definitions require an environment $\theta$, called an interpretation, to give meaning to type variables. Concretely, an interpretation is a partial map from type variables to sets of terms. An interpretation $\theta$ has the constraint that for every type variable $\alpha \in \operatorname{dom}(\theta), \theta(\alpha)$ is a reducibility candidate $\mathrm{C}$, which, in our setting, means that all terms in $\theta(\alpha)$ are (erased) values. The set of all reducibility candidates is denoted by Candidates $\subseteq 2^{\text {Terms }}$, and an interpretation $\theta$ is therefore a partial map in $\mathcal{V} \mapsto$ Candidates.

When the interpretation has no influence on the definition, we may omit it. For instance, for every $\theta \in\left(\mathcal{V} \mapsto\right.$ Candidates), we have $\llbracket N a t \rrbracket_{\mathrm{v}}^{\theta}=\{$ zero, $\operatorname{succ}($ zero $), \operatorname{succ}(\operatorname{succ}($ zero $)), \ldots\}$, so we can just denote this set by $\llbracket \mathrm{Nat} \rrbracket_{\mathrm{v}}$.

By construction, $\llbracket \tau \rrbracket_{\mathrm{v}}^{\theta}$ only contains (erased) values (of type $\tau$ ), while $\llbracket \tau \rrbracket_{\mathrm{t}}^{\theta}$ contains (erased) terms that reduce to a value in $\llbracket \tau \rrbracket_{\mathrm{v}}^{\theta}$. For example, a term in $\llbracket N a t \rightarrow \mathrm{Nat} \rrbracket_{\mathrm{t}}^{\theta}$ is not only normalizing as a term of its own, but also normalizes whenever applied to a value in $\llbracket N a t \rrbracket_{\mathrm{v}}^{\theta}$.

The type $\{x: \tau \mid b\}$ represents the values $v$ of type $\tau$ for which $b[x \mapsto v]$ evaluates to true. We use this type as a building block for writing specifications (pre and postconditions).

The type $\forall x: \tau_{1} . \tau_{2}$ represents the values that are in the intersection of the types $\tau_{2}[x \mapsto a]$ when $a$ ranges over values of type $\tau_{1}$. This type differs from $\Pi x: \tau_{1}, \tau_{2}$ in the sense that a value in $\forall x: \tau_{1}, \tau_{2}$ belongs to every $\tau_{2}[x \mapsto a]$ for $a$ in $\tau_{1}$, while a value in $\Pi x: \tau_{1}, \tau_{2}$ is a function that, 


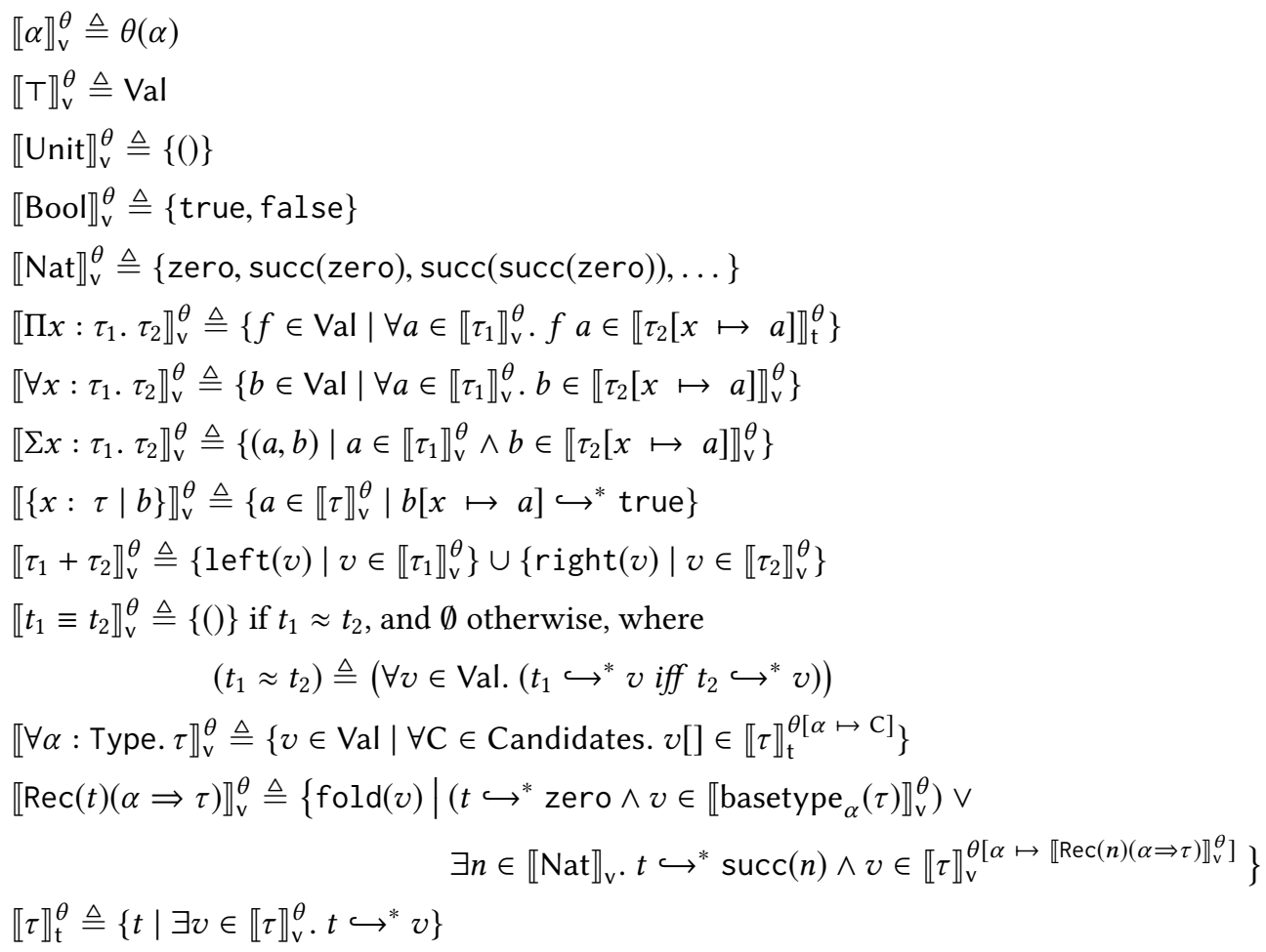

$$
\begin{aligned}
& \text { basetype }_{\alpha}\left(\Sigma x: \tau_{1} \cdot \tau_{2}\right) \triangleq \Sigma x \text { : basetype }{ }_{\alpha}\left(\tau_{1}\right) \text {. basetype }{ }_{\alpha}\left(\tau_{2}\right) \\
& \text { basetype }_{\alpha}\left(\tau_{1}+\tau_{2}\right) \triangleq \text { basetype }_{\alpha}\left(\tau_{1}\right)+\text { basetype }_{\alpha}\left(\tau_{2}\right) \\
& \text { basetype }_{\alpha}(\tau) \triangleq \text { if } \alpha \in \operatorname{fv}(\tau) \text { then } T \text { else } \tau
\end{aligned}
$$

Fig. 13. Definition of reducibility for values and for terms for each type. The function basetype() is an auxiliary function, used in the base case of the definition for recursive types.

when applied to some $a$ in $\tau_{1}$, produces a value in $\tau_{2}[x \mapsto a]$. From a value $b$ in $\forall x: \tau_{1} . \tau_{2}$, we can build a value in $\Pi x: \tau_{1} . \tau_{2}$ (namely, $\lambda x . b$ ), while the other way around is not always possible.

The sum type $\tau_{1}+\tau_{2}$ represents values that are either of the form left $(v)$ where $v$ is a reducible value of $\tau_{1}$, or of the form right $(v)$ where $v$ is a reducible value of $\tau_{2}$.

The set of reducible values for the equality type $\llbracket t_{1} \equiv t_{2} \rrbracket_{\mathrm{v}}^{\theta}$ makes use of a notion of equivalence on terms which is based on operational semantics. More specifically, we say that $t_{1}$ and $t_{2}$ are equivalent, denoted $t_{1} \approx t_{2}$, if for every value $v$, we have $t_{1} \hookrightarrow^{*} v$ iff $t_{2} \hookrightarrow^{*} v$. Note that this equivalence relation is defined even if we do not know anything about the types of terms $t_{1}$ and $t_{2}$, and it ensures that if one of the terms reduces to a value, then so does the other.

The type $\forall \alpha$ : Type. $\tau$ is the polymorphic type from System F. The set $\llbracket \forall \alpha:$ Type. $\tau \rrbracket_{v}^{\theta}$ is defined by using the environment $\theta$ to bind the type variable $\alpha$ to an arbitrary reducibility candidate.

We use the recursive type $\operatorname{Rec}(n)(\alpha \Rightarrow \tau)$ as a building block for representing data structures such as lists of streams. The definition of reducibility for the recursive type makes use of an auxiliary 
function basetype() that can be seen as an (upper) approximation of the recursive type. Note that basetype $_{\alpha}(\tau)$ (defined at the bottom of Figure 13) removes the type variable $\alpha$ from $\tau$.

Our reducibility definition respects typical lemmas that are needed to prove the soundness of typing rules, such as the following substitution lemma (see [Girard 1971] for the lemma on System F), which we have formally proven (see also Section 6 below).

Lemma 4.1. Let $\tau_{1}$ and $\tau_{2}$ be two types, and let $\alpha$ be a type variable that may appear in $\tau_{1}$ but not in $\tau_{2}$. Let $\theta$ be a type interpretation. Then, we have:

$$
\llbracket \tau_{1} \rrbracket_{v}^{\theta\left[\alpha \mapsto \llbracket \tau_{2} \rrbracket_{v}^{\theta}\right]}=\llbracket \tau_{1}\left[\alpha \mapsto \tau_{2}\right]_{v}^{\theta}
$$

4.1.1 Well-Foundedness of the Reduciblity Definition. We can show that the definition given Figure 13 is well-founded by defining a lexicographic measure (typeNodes $(\tau)$, index $(\tau)) \in \mathbb{N} \times$ Terms on types $\tau$. The function typeNodes $(\tau)$ returns the size of the syntactic tree of type $\tau$, ignoring the terms that appear inside. This size roughly corresponds to the number of (top-level) type constructors in the tree of $\tau$. For example, typeNodes $(\{x: \tau \mid p\})=1+\operatorname{typeNodes}(\tau)$ (ignoring $p$ ), typeNodes $\left(\tau_{1}+\tau_{2}\right)=1+\operatorname{typeNodes}\left(\tau_{1}\right)+\operatorname{typeNodes}\left(\tau_{2}\right)$, and typeNodes $\left(t_{1} \equiv t_{2}\right)=0$. Ignoring the size of terms inside types ensures that given a type $\tau$, a term variable $x$, and a term $a$, we have: $\operatorname{typeNodes}(\tau[x \mapsto a])=\operatorname{typeNodes}(\tau)$. As a result, the measure typeNodes $(\tau)$ in the definition of reducibility decreases for indexed types such as $\Sigma x: \tau_{1}, \tau_{2}$ or $\Pi x: \tau_{1}, \tau_{2}$.

The number typeNodes $(\tau)$ decreases in every case of Figure 13, except for recursive types $\operatorname{Rec}(t)(\alpha \Rightarrow \tau)$ where the measure stays the same in the recursive call to the denotation on $\operatorname{Rec}(n)(\alpha \Rightarrow \tau)$ with $t \hookrightarrow^{*}$ succ $(n)$. This is where we use the second component of the lexicographic measure, index $(\tau)$. We define index $(\operatorname{Rec}(t)(\alpha \Rightarrow \tau))=t$ and index $(\tau)=\operatorname{err}$ for every other type. Then, given $t_{1}, t_{2} \in$ Terms, we consider $t_{1}$ to be (strictly) smaller than $t_{2}$ if there exist $v_{1}, v_{2} \in \llbracket \mathrm{Nat} \rrbracket_{\mathrm{v}}$, such that $t_{1} \hookrightarrow^{*} v_{1}, t_{2} \hookrightarrow^{*} v_{2}$, and $v_{1}$ is strictly smaller than $v_{2}$ when seen as a natural number. Therefore, the second component index $(\operatorname{Rec}(n)(\alpha \Rightarrow \tau))=n$ is strictly smaller than index $(\operatorname{Rec}(t)(\alpha \Rightarrow \tau))=t$ in the definition (because $t \hookrightarrow^{*} \operatorname{succ}(n)$ ), which ensures that the overall lexicographic measure decreases.

\subsection{Reduciblity for Open Terms}

Having defined reducibility for closed terms, we now define what it means for a term $t$ with free term and type variables to be reducible for a type $\tau$. Informally, we want to ensure that for every interpretation of the type variables, and for every substitution of values for the term variables, the term $t$ reduces in a finite number of steps to a value in type $\tau$. This is formalized by a (semantic) typing relation $\Theta ; \Gamma \mid F_{\text {Red }} t: \tau$ which is defined as follows.

First, a context $\Theta ; \Gamma$ is made of a finite set $\Theta \subseteq \mathcal{V}$ of type variables and of a sequence $\Gamma$ of pairs in $\mathcal{V} \times$ Type. The domain of $\Gamma$, denoted $\operatorname{dom}(\Gamma)$ is the list of variables (in $\mathcal{V}$ ) appearing in the left-hand-sides of the pairs. We implicitly assume throughout the paper that all variables appearing in the domains are distinct. This enables us to use $\Gamma$ as a partial map from $\mathcal{V}$ to Type. We use a sequence to represent $\Gamma$ as the order of variables is important, since a variable may have a (dependent) type which refers to previous variables in the context.

Given a partial map $\gamma \in \mathcal{V} \mapsto$ Terms, we write $\gamma(t)$ for the term $t$ where every variable $x$ is replaced by $\gamma(x)$. We use the same notation $\gamma(\tau)$ for applying a substitution to a type $\tau$.

Given a context $\Theta ; \Gamma$, a reducible substitution for $\Theta ; \Gamma$ is a pair of partial maps $\theta \in \mathcal{V} \mapsto$ Candidates and $\gamma \in \mathcal{V} \mapsto$ Terms where: $\operatorname{dom}(\theta)=\Theta, \operatorname{dom}(\gamma)=\operatorname{dom}(\Gamma)$, and $\forall x \in \operatorname{dom}(\Gamma), \gamma(x) \in \llbracket \gamma(\Gamma(x)) \rrbracket_{\mathrm{v}}^{\theta}$.

Note that the substitution $\gamma$ is also applied to the type $\Gamma(x)$, since $\Gamma(x)$ may be a dependent type with free term variables. The set of all pairs of reducible substitutions for $\Theta ; \Gamma$ is denoted $\llbracket \Theta ; \Gamma \rrbracket_{\mathrm{v}}$. 
Finally, given a context $\Theta ; \Gamma$, a term $t$ and a type $\tau$, we say that $\Theta ;\left.\Gamma\right|_{\text {Red }} t: \tau$ holds when for every pair of substitutions $\theta, \gamma$ for the context $\Theta ; \Gamma, \gamma(t)$ belongs the reducible values at type $\gamma(\tau)$. Formally, $\Theta ; \Gamma \mid \operatorname{Red} t: \tau$ is defined to hold when:

$$
\forall \theta, \gamma \in \llbracket \Theta ; \Gamma \rrbracket_{\mathrm{v}} \cdot \gamma(t) \in \llbracket \gamma(\tau) \rrbracket_{\mathrm{t}}^{\theta}
$$

Our bidirectional type checking and inference algorithm in Section 5 is a sound (even if incomplete) procedure to check $\Theta ; \Gamma \mid F_{\operatorname{Red}} t: \tau$.

\subsection{Recursive Types}

We explain in this section how to interpret the type $\operatorname{Rec}(n)(\alpha \Rightarrow \tau)$ (see reducibility definition in Figure 13) and how the Stream[X] and List $[\mathrm{X}]$ types represent streams and lists.

4.3.1 Infinite Streams. For a natural number $n$, consider the type $S_{n} \triangleq \operatorname{Stream}_{n}[\mathrm{Nat}] \triangleq$ $\operatorname{Rec}(n)(\alpha \Rightarrow$ Nat $\times($ Unit $\rightarrow \alpha))$. Let us first see what $S_{n}$ represents for small values of $n$. As a shortcut, we use the notations $0,1,2, \ldots$ for zero, $\operatorname{succ}(z e r o), \operatorname{succ}(\operatorname{succ}(z e r o)), \ldots$

The definition $\llbracket S_{0} \rrbracket_{\mathrm{V}}$ refers to basetype ${ }_{\alpha}(\mathrm{Nat} \times($ Unit $\rightarrow \alpha))$, which is Nat $\times \mathrm{T}$ by definition. This means that $\llbracket S_{0} \rrbracket_{\mathrm{v}}$ is the set of values of the form fold $((a, v))$, where $a \in \llbracket \mathrm{Nat} \rrbracket_{\mathrm{v}}$, and $v \in \mathrm{Val}$.

By unrolling the definition, we get that $\llbracket S_{1} \rrbracket_{v}$ is the set of values of the form fold $(v)$ where $v$ is in $\llbracket$ Nat $\times($ Unit $\rightarrow \alpha) \rrbracket_{\mathrm{v}}^{\left[\alpha \mapsto \llbracket S_{0} \rrbracket_{\mathrm{v}}\right]}$, which is the same (by Lemma 4.1) as $\llbracket$ Nat $\times\left(\right.$ Unit $\left.\rightarrow S_{0}\right) \rrbracket_{\mathrm{v}}$. Therefore, $\llbracket S_{1} \rrbracket_{\mathrm{v}}$ is the set of values of the form fold $(a, f)$ where $a \in \llbracket N a t \rrbracket_{\mathrm{v}}$ and $f \in \llbracket$ Unit $\rightarrow S_{0} \rrbracket_{\mathrm{v}}$. This means that when it is applied to ()$, f$ terminates and returns a value in $\llbracket S_{0} \rrbracket_{\mathrm{v}}^{\theta}$. Similarly, $\llbracket S_{2} \rrbracket_{\mathrm{v}}$ is the set of values of the form fold $(a, f)$ where $n \in \llbracket N a t \rrbracket_{\mathrm{v}}$ and $f \in \llbracket U n i t \rightarrow S_{1} \rrbracket_{\mathrm{v}}$.

To summarize, we can say that for every $n \in \llbracket N a t \rrbracket_{v}, S_{n}$ represents values of the language that behave as streams of natural numbers, as long as they are unfolded at most $n+1$ times. This matches the property we mentioned in Section 2, as $S_{n}$ represents the streams that are $n+1$-non-diverging. We can show that as $n$ grows, $S_{n}$ gets more and more constraints: $\llbracket S_{0} \rrbracket_{\mathrm{v}} \supseteq \llbracket S_{1} \rrbracket_{\mathrm{v}} \supseteq \llbracket S_{2} \rrbracket_{\mathrm{v}} \supseteq \ldots$ In the limit, a value $v \in \llbracket \forall n:$ Nat. $S_{n} \rrbracket_{\mathrm{v}}$ (which is in every $S_{n}$ for $n \in \llbracket N a t \rrbracket_{\mathrm{v}}$ ), represents a stream of natural numbers, that, regardless of the number of times it is unfolded, does not diverge, i.e. a non-diverging stream. Equivalently, we have $v \in \llbracket$ Stream[Nat $] \rrbracket_{v}$.

4.3.2 Finite Lists. Types of the form $\operatorname{Rec}(\alpha \Rightarrow \tau)$ can also be used to represent finite data structures such as lists. We let List $_{n}[\mathrm{X}]$ be a notation for $\operatorname{Rec}(n)(\alpha \Rightarrow$ Unit $+\mathrm{X} \times \alpha)$, so that:

$$
\text { List }[\mathrm{X}] \triangleq \forall n: \text { Nat. List }{ }_{n}[\mathrm{X}] \text {. }
$$

Here are some examples to show how lists are encoded:

- The empty list is fold(left()),

- A list with one element $n$ is fold(right $(n$, fold $(\operatorname{left}())))$,

- Given an element $n$ and a list $l$, we can construct the list $n:: l$ by writing: fold $(\operatorname{right}(n, l))$.

Let us now see why List $[X]$ represents the type of all finite lists of elements in $X$. The first thing to note is that given $n \in \llbracket N a t \rrbracket_{\mathrm{v}}$, List ${ }_{n}[\mathrm{X}]$ does not represent the lists of size $n$. For instance, we know that $\llbracket$ List $_{0}[X] \rrbracket_{v}$ is the set of values of the form fold $(v)$ where $v \in \llbracket$ basetype ${ }_{\alpha}($ Unit $+X \times \alpha) \rrbracket_{v}$, i.e. $v \in \llbracket T \rrbracket_{v}=$ Val. Therefore, List $_{0}[X]$ contains lists of all sizes (and also all values that do not represent lists, such as fold(zero) or fold $(\lambda x .(()))$.

Instead, List ${ }_{n}[\mathrm{X}]$ can be understood as the values that, as long as they are unfolded no more than $n$ times, behave as lists. As for streams, we have: $\llbracket$ List $_{0}[X] \rrbracket_{v} \supseteq \llbracket$ List $_{1}[X] \rrbracket_{v} \supseteq \llbracket$ List $_{2}[X] \rrbracket_{v} \supseteq \ldots$ where the monotonicity follows because $\alpha$ only appears in positive positions in the definitions of the recursive types for streams and lists. In the limit, we can show that List $[X]$ contains all finite lists, and nothing more. 
Lemma 4.2. Let $v \in \quad \mathrm{Val}$ be a value and $\mathrm{X}$ be some type. Then, $v \in \llbracket$ List $[\mathrm{X}] \rrbracket_{v}$ if and only if there exists $k \geq 0$ and $a_{1}, \ldots, a_{k} \in \llbracket \mathrm{X} \rrbracket_{v}$ such that $v=$ fold $\left(\operatorname{right}\left(a_{1}, \ldots\right.\right.$ fold $\left(\operatorname{right}\left(a_{k}\right.\right.$, fold $\left.\left.\left.\left.(\operatorname{left}())\right)\right) \ldots\right)\right)$.

It may seem surprising that the type of streams $\operatorname{Rec}(\alpha \Rightarrow \mathrm{X} \times($ Unit $\rightarrow \alpha))$ contains infinite streams while the type of lists $\operatorname{Rec}(\alpha \Rightarrow$ Unit $+\mathrm{X} \times \alpha)$ only contains finite lists. The reason is that, in a call-by-value language, a value representing an infinite list would need to have an infinite syntax tree, with infinitely many fold()'s (which is not possible). On the other hand, we can represent infinite streams by hiding recursion underneath a lambda term as shown in Section 2.

\section{A BIDIRECTIONAL TYPE-CHECKING ALGORITHM}

In this section, we give procedures for inferring a type $\tau$ for a term $t$ in a context $\Theta ; \Gamma$, denoted $\Theta ; \Gamma \vdash t \Uparrow \tau$, as well as for checking that the type of a term $t$ is $\tau$, denoted $\Theta ; \Gamma \vdash t \Downarrow \tau$. We introduce rules of our procedures throughout this section; the full set of rules is given in figures 14 and 15 .

Our inference and checking rules give rise to conditions of the form $\Theta ; \Gamma \vdash t_{1} \equiv t_{2}$. We call such checks verification conditions (in the rules, they are boxed and appear in blue color). The $\equiv$ sign is part of the judgment form, and does not describe a formula. We rely on an external solver to perform these checks, and assume that when the verification condition is considered valid by the solver, then: $\forall \theta, \gamma \in \llbracket \Theta ; \Gamma \rrbracket_{v} \cdot \gamma\left(\operatorname{erase}\left(t_{1}\right)\right) \approx \gamma\left(\operatorname{erase}\left(t_{2}\right)\right)$. This is an equivalent way of saying that $\llbracket \gamma\left(\operatorname{erase}\left(t_{1}\right)\right) \equiv \gamma\left(\operatorname{erase}\left(t_{2}\right)\right) \rrbracket_{\mathrm{v}}^{\theta}$ is non-empty. Under these conditions, we have the following theorem.

Theorem 5.1 (Soundness of the Bidirectional Type-Checker). If $\Theta ; \Gamma \vdash t \Uparrow \tau$ holds or if $\Theta ; \Gamma \vdash t \Downarrow \tau$ holds, then $\Theta ;\left.\operatorname{erase}(\Gamma)\right|_{\mathrm{Red}} \operatorname{erase}(t)$ : erase $(\tau)$ holds.

\subsection{Annotated Terms}

In order to guide our type-checking algorithm, we require terms to be annotated. We give in Figure 16 the grammar for annotated terms. The term inst $\left(t_{1}, t_{2}\right)$ is used to instantiate a term $t_{1}$ which has a type of the form $\forall x: \tau_{2} . \tau$ to a particular term $t_{2}$ of type $\tau_{2}$, in the (Infer Forall Instantiation) type inference rule of Figure 14. The term unfold_pos $t_{1}$ in $t_{2}$ is an annotated variant of unfold $t_{1}$ in $t_{2}$ (see rules (Infer Unfold) and (Infer Unfold Positive) in Figure 14). We discuss the difference between these rules in Section 5.6.

The type Let $x=t_{2}$ in $\tau$ represents the type $\tau$ where the variable $x$ is bound to $t_{2}$ by using let's in each term that appears in $\tau$. The formal definition is given in Section 5.

Annotations such as $\lambda x: \tau$. $t$ or inst $\left(t_{1}, t_{2}\right)$ have no runtime influence and are erased (respectively to $\lambda x . t$ and $\left.t_{1}\right)$. We write erase $(t)$ to refer to the erasure of $t$, where every annotation has been erased. The full definition is given in the long version of our paper [Hamza et al. 2019].

When a type $\tau$ has annotated terms inside, we write erase $(\tau)$ to erase their annotations. For instance erase $(\{x: \tau \mid b\})$ refers to $\{x: \tau \mid \operatorname{erase}(b)\}$. Moreover, for a context $\Gamma$, we write erase $(\Gamma)$ to refer to the context $\Gamma$ where each type $\tau$ has been replaced by erase $(\tau)$.

\subsection{Contracts and Measures}

The syntax we support in our verification tool translates into our core calculus presented above. In our tool we support named functions with contracts and measures which are desugared into fix terms. To compare natural numbers and express the fact that measures decrease, we use functions ' $<$ ', '<=' and '==' on natural numbers. These functions can be defined using the recursor rec (see the long version of our paper for definitions [Hamza et al. 2019]).

Figure 17 shows how, thanks to refinement types, the fix term can encode recursive functions (such as the one given in Section 2) that feature user-defined pre- and post-conditions and whose 


$$
\begin{aligned}
& \frac{\Gamma(x)=\tau}{\Theta ; \Gamma \vdash x \Uparrow \tau} \text { (Infer Var) } \overline{\Theta ; \Gamma \vdash \text { true } \Uparrow \text { Bool }} \text { (Infer True) } \overline{\Theta ; \Gamma \vdash \text { false } \Uparrow \text { Bool }} \text { (Infer False) } \\
& \overline{\Theta ; \Gamma \vdash() \Uparrow \text { Unit }} \text { (Infer Unit) } \frac{\Theta ; \Gamma \vdash \text { zero } \Uparrow N a t}{(\text { Infer Zero) }} \frac{\Theta ; \Gamma \vdash t \Downarrow N \text { Nat }}{\Theta ; \Gamma \vdash \operatorname{succ}(t) \Uparrow N a t} \text { (Infer Succ) } \\
& \frac{\Theta ; \Gamma \vdash t_{1} \Downarrow \text { Bool } \Theta ; \Gamma, p: t_{1} \equiv \text { true } \vdash t_{2} \Uparrow \tau_{2} \quad \Theta ; \Gamma, p: t_{1} \equiv \text { false } \vdash t_{3} \Uparrow \tau_{3}}{\Theta ; \Gamma \vdash \text { if } t_{1} \text { then } t_{2} \text { else } t_{3} \Uparrow \text { If } t_{1} \text { Then } \tau_{2} \text { Else } \tau_{3}} \text { (Infer If) } \\
& \frac{\Theta ; \Gamma \vdash t \Downarrow \tau_{1}}{\Theta ; \Gamma \vdash \operatorname{left}\left[\tau_{1}+\tau_{2}\right](t) \Uparrow \tau_{1}+\tau_{2}} \text { (Infer Left) } \frac{\Theta ; \Gamma \vdash t \Downarrow \tau_{2}}{\Theta ; \Gamma \vdash \operatorname{right}\left[\tau_{1}+\tau_{2}\right](t) \Uparrow \tau_{1}+\tau_{2}} \text { (Infer Right) } \frac{\Theta ; \Gamma \vdash t \Uparrow \tau}{\Theta ; \Gamma \vdash \operatorname{size}(t) \Uparrow N a t} \text { (Infer Size) } \\
& \frac{\Theta ; \Gamma \vdash t_{n} \Downarrow \mathrm{Nat} \quad \Theta ; \Gamma, p: t_{n} \equiv \text { zero } t_{0} \Uparrow \tau_{1} \quad \Theta ; \Gamma, n: \text { Nat, } p: t_{n} \equiv \operatorname{succ}(n) \vdash t_{s} \Uparrow \tau_{2}}{\Theta ; \Gamma \vdash \operatorname{match}\left(t_{n}, t_{0}, n \Rightarrow t_{s}\right) \Uparrow \operatorname{Match}\left(t_{n}, \tau_{1}, n \Rightarrow \tau_{2}\right)} \text { (Infer Match) } \\
& \frac{\Theta ; \Gamma \vdash t \Uparrow \tau_{1}+\tau_{2} \quad \Theta ; \Gamma, x: \tau_{1}, p: t \equiv \operatorname{left}(x) \vdash t_{1} \Uparrow \tau_{1}^{\prime} \quad \Theta ; \Gamma, x: \tau_{2}, p: t \equiv \operatorname{right}(x) \vdash t_{2} \Uparrow \tau_{2}^{\prime}}{\Theta ; \Gamma \vdash \text { either_match }\left(t, x \Rightarrow t_{1}, x \Rightarrow t_{2}\right) \Uparrow \text { Either_Match }\left(t, x \Rightarrow \tau_{1}^{\prime}, x \Rightarrow \tau_{2}^{\prime}\right)} \text { (Infer Either Match) } \\
& \Theta ; \Gamma \vdash t_{n} \Downarrow \text { Nat } \quad \Theta ; \Gamma \vdash t_{0} \Downarrow \tau[n \mapsto \text { zero }] \\
& \frac{\Theta ; \Gamma, n: \text { Nat, } y: \text { Unit } \rightarrow \tau, p: y \equiv \lambda u: \text { Unit. } \operatorname{rec}[n \rightarrow \tau]\left(n, t_{0},(n, y) \Rightarrow t_{s}\right) \vdash t_{s} \Downarrow \tau[n \mapsto \operatorname{succ}(n)]}{\Theta ; \Gamma \vdash \operatorname{rec}[n \Rightarrow \tau]\left(t_{n}, t_{0},(n, y) \Rightarrow t_{s}\right) \Uparrow \text { Let } n=t_{n} \text { in } \tau} \text { (Infer Rec) } \\
& n \notin \mathrm{fv}(\operatorname{erase}(t)) \\
& \Theta ; \Gamma, n: \text { Nat, } y: \text { Unit } \rightarrow \forall m:\{m: \text { Nat } \mid m<n\} . \tau[n \mapsto m] \\
& \begin{array}{c}
p: y \equiv \lambda u: \text { Unit. fix }[n \Rightarrow \tau]((n, y) \Rightarrow t) \quad \vdash t \Downarrow \tau \\
\text { (Infer Fix) }
\end{array} \\
& \frac{\Theta ; \Gamma \vdash t_{1} \Uparrow \tau_{1} \Theta ; \Gamma \vdash t_{2} \Uparrow \tau_{2}}{\Theta ; \Gamma \vdash\left(t_{1}, t_{2}\right) \Uparrow \Sigma x: \tau_{1} \cdot \tau_{2}} \text { (Infer Pair) } \frac{\Theta ; \Gamma \vdash t_{1} \Uparrow \tau_{1} \quad \Theta ; \Gamma, x: \tau_{1}, p: x \equiv t_{1} \vdash t_{2} \Uparrow \tau_{2}}{\Theta ; \Gamma \vdash \operatorname{let} x=t_{1} \text { in } t_{2} \Uparrow \text { Let } x=t_{1} \text { in } \tau_{2}} \text { (Infer Let) } \\
& \frac{\Theta ; \Gamma \vdash t \Uparrow \Sigma x: \tau_{1} \cdot \tau_{2}}{\Theta ; \Gamma \vdash \pi_{1} t \Uparrow \tau_{1}} \text { (Infer Proj1) } \quad \frac{\Theta ; \Gamma \vdash t \Uparrow \Sigma x: \tau_{1} \cdot \tau_{2}}{\Theta ; \Gamma \vdash \pi_{2} t \Uparrow \text { Let } x=\pi_{1} t \text { in } \tau_{2}} \text { (Infer Proj2) }
\end{aligned}
$$

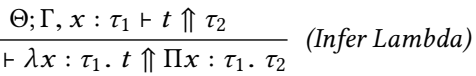

$$
\begin{aligned}
& \frac{\Theta ; \Gamma \vdash t_{1} \Uparrow \Pi x: \tau_{2} \cdot \tau \quad \Theta ; \Gamma \vdash t_{2} \Downarrow \tau_{2}}{\Theta ; \Gamma \vdash t_{1} t_{2} \Uparrow \text { Let } x=t_{2} \text { in } \tau} \text { (Infer App) } \\
& \frac{\Theta, \alpha ; \Gamma \vdash t \Uparrow \tau}{\Theta ; \Gamma \vdash \Lambda \alpha . t \Uparrow \forall \alpha: \text { Type. } \tau} \text { (Infer Type Abs) } \frac{\Theta ; \Gamma \vdash t \Uparrow \forall \alpha: \text { Type. } \tau_{1}}{\Theta ; \Gamma \vdash t\left[\tau_{2}\right] \Uparrow \tau_{1}\left[\alpha \mapsto \tau_{2}\right]} \text { (Infer Type App) } \\
& \frac{\Theta ; \Gamma \vdash t_{1} \Uparrow \forall x: \tau_{2} \cdot \tau \quad \Theta ; \Gamma \vdash t_{2} \Downarrow \tau_{2}}{\Theta ; \Gamma \vdash \operatorname{inst}\left(t_{1}, t_{2}\right) \Uparrow \text { Let } x=t_{2} \text { in } \tau} \text { (Infer Forall Instantiation) } \\
& \Theta ; \Gamma \vdash n \Downarrow \text { Nat } \quad \Theta ; \Gamma, p: n \equiv \text { zero } t \downarrow \Downarrow \text { basetype }_{\alpha}(\tau) \\
& \frac{\Theta ; \Gamma ; n^{\prime}: \text { Nat, } p: n \equiv \operatorname{succ}\left(n^{\prime}\right) \vdash t \Downarrow \tau\left[\alpha \mapsto \operatorname{Rec}\left(n^{\prime}\right)(\alpha \Rightarrow \tau)\right]}{\Theta ; \Gamma \vdash \operatorname{fold}[\operatorname{Rec}(n)(\alpha \Rightarrow \tau)](t) \Uparrow \operatorname{Rec}(n)(\alpha \Rightarrow \tau)} \text { (Infer Fold) } \\
& \Theta ; \Gamma \vdash t_{1} \Uparrow \operatorname{Rec}(n)(\alpha \Rightarrow \tau) \quad \Theta ; \Gamma, x: \text { basetype }_{\alpha}(\tau), p_{1}: t_{1} \equiv \text { fold }(x), p_{2}: n \equiv \text { zero } t_{2} \Uparrow \tau^{\prime} \\
& \Theta ; \Gamma, x: \tau[\alpha \mapsto \operatorname{Rec}(\operatorname{pred}(n))(\alpha \Rightarrow \tau)], p: t_{1} \equiv \operatorname{fold}(x) \vdash t_{2} \Uparrow \tau^{\prime} \\
& \Theta ; \Gamma \vdash \text { unfold } t_{1} \text { in } x \Rightarrow t_{2} \Uparrow \tau^{\prime} \\
& \Theta ; \Gamma \vdash t_{1} \Uparrow \operatorname{Rec}(n)(\alpha \Rightarrow \tau) \quad \Theta ; \Gamma \vdash \text { lessThan } 0 n \equiv \text { true } \\
& \frac{\Theta ; \Gamma, x: \tau[\alpha \mapsto \operatorname{Rec}(\operatorname{pred}(n))(\alpha \Rightarrow \tau)], p: t_{1} \equiv \operatorname{fold}(x) \vdash t_{2} \Uparrow \tau^{\prime}}{\Theta ; \Gamma \vdash \text { unfold } t_{1} \text { in } x \Rightarrow t_{2} \Uparrow \tau^{\prime}} \text { (Infer Unfold Positive) } \\
& \frac{\Theta ; \Gamma \vdash \text { true } \equiv \text { false }}{\Theta ; \Gamma \vdash \operatorname{err}[\tau] \Uparrow \tau} \text { (Infer Err) } \frac{\Theta ; \Gamma \vdash t_{1} \equiv t_{2}}{\Theta ; \Gamma \vdash \operatorname{refl}\left[t_{1}, t_{2}\right] \Uparrow t_{1} \equiv t_{2}} \text { (Infer Refl) } \\
& \frac{\Theta ; \Gamma \vdash t \Uparrow\{x: \tau \mid p\}}{\Theta ; \Gamma \vdash t \Uparrow \tau} \text { (Infer Drop Refinement) }
\end{aligned}
$$

Fig. 14. $\Theta ; \Gamma \vdash t \Uparrow \tau$ infers a type $\tau$ for $t$ in context $\Theta ; \Gamma$ based on the shape of $t$. The (Infer Drop Refinement) rule is applied with low priority, only if no other rule is applicable, keeping type checking deterministic. 


$$
\begin{gathered}
\frac{\Theta ; \Gamma \vdash t_{1} \Downarrow \text { Bool } \quad \Theta ; \Gamma, p: t_{1} \equiv \operatorname{true} \vdash t_{2} \Downarrow \tau \quad \Theta ; \Gamma, p: t_{1} \equiv \text { false } \vdash t_{3} \Downarrow \tau}{\Theta ; \Gamma \vdash \text { if } t_{1} \text { then } t_{2} \text { else } t_{3} \Downarrow \tau} \text { (Check If) } \\
\frac{\Theta ; \Gamma \vdash t_{n} \Downarrow \text { Nat } \quad \Theta ; \Gamma, p: t_{n} \equiv \operatorname{zero} \vdash t_{0} \Downarrow \tau \quad \Theta ; \Gamma, n: \text { Nat, } p: t_{n} \equiv \operatorname{succ}(n) \vdash t_{s} \Downarrow \tau}{\Theta ; \Gamma \vdash \operatorname{match}\left(t_{n}, t_{0}, n \Rightarrow t_{s}\right) \Downarrow \tau} \text { (CheckMatch) }
\end{gathered}
$$

$\Theta ; \Gamma \vdash t \Uparrow \tau_{1}+\tau_{2} \quad \Theta ; \Gamma, x: \tau_{1}, p: t \equiv \operatorname{left}(x) \vdash t_{1} \Downarrow \tau \quad \Theta ; \Gamma, x: \tau_{2}, p: t \equiv \operatorname{right}(x) \vdash t_{2} \Downarrow \tau$

$\Theta ; \Gamma \vdash$ either_match $\left(t, x \Rightarrow t_{1}, x \Rightarrow t_{2}\right) \Downarrow \tau$ (Check Either Match)

$\frac{\Theta ; \Gamma \vdash t_{1} \Uparrow \tau_{1} \quad \Theta ; \Gamma, x: \tau_{1}, p: x \equiv t_{1} \vdash t_{2} \Downarrow \tau}{\Theta ; \Gamma \vdash \operatorname{let} x=t_{1} \text { in } t_{2} \Downarrow \tau}$ (Check Let) $\quad \frac{\Theta ; \Gamma, x: \text { Nat } \vdash t[x] \Downarrow \tau}{\Theta ; \Gamma \vdash t \Downarrow \forall x: \text { Nat. } \tau}$ (Check Forall)

$\frac{\Theta ; \Gamma, x: \tau_{1} \vdash t x \Downarrow \tau_{2}}{\Theta ; \Gamma \vdash t \Downarrow \Pi x: \tau_{1} \cdot \tau_{2}}$ (Check Pi) $\frac{\Theta ; \Gamma \vdash \pi_{1} t \Downarrow \tau_{1} \quad \Theta ; \Gamma, x: \tau_{1}, p: x \equiv \pi_{1} t \vdash \pi_{2} t \Downarrow \tau_{2}}{\Theta ; \Gamma \vdash t \Downarrow \Sigma x: \tau_{1} \cdot \tau_{2}}$ (Check Sigma)

$$
\frac{\Theta ; \Gamma \vdash t \Downarrow \tau \quad \Theta ; \Gamma, x: \tau, p: x \equiv t \vdash b \equiv \text { true }}{\Theta ; \Gamma \vdash t \Downarrow\{x: \tau \mid b\}} \text { (Check Refinement) }
$$

$$
\begin{aligned}
& \frac{\Theta, \alpha ; \Gamma \vdash t[\alpha] \Downarrow \tau}{\Theta ; \Gamma \vdash t \Downarrow \forall \alpha: \text { Type. } \tau} \text { (Check Type Abs) } \quad \frac{\Theta ; \Gamma \vdash t \Uparrow \operatorname{Rec}\left(n_{2}\right)(\alpha \Rightarrow \tau) \quad \Theta ; \Gamma \vdash n_{1} \equiv n_{2}}{\Theta ; \Gamma \vdash t \Downarrow \operatorname{Rec}\left(n_{1}\right)(\alpha \Rightarrow \tau)} \text { (Check Recursive) } \\
& \frac{v \in \mathrm{Val}}{\Theta ; \Gamma \vdash v \Downarrow T} \text { (Check Top 1) } \frac{\Theta ; \Gamma \vdash t \Uparrow \tau}{\Theta ; \Gamma \vdash t \Downarrow \top} \text { (Check Top 2) } \frac{\Theta ; \Gamma \vdash t \Uparrow \tau}{\Theta ; \Gamma \vdash t \Downarrow \tau} \text { (Check Reflexive) }
\end{aligned}
$$

Fig. 15. $\Theta ; \Gamma \vdash t \Downarrow \tau$ checks that term $t$ indeed has type $\tau$ under context $\Theta ; \Gamma$. When multiple rules are applicable, they are applied from a priority order from top to bottom, left to right. The (Check Forall) rule can be generalized to (non-empty) types other than Nat, but we only need it for Nat (in type $\operatorname{Rec}(\alpha \Rightarrow \tau)$ ).

$$
\begin{aligned}
& t::=x|() \quad| \quad \lambda x: \tau \cdot t|t t \quad| \quad(t, t)\left|\pi_{1} t\right| \pi_{2} t \quad|\quad \operatorname{inst}(t, n)| \\
& \operatorname{left}[\tau+\tau](t)|\operatorname{right}[\tau+\tau](t)| \text { either_match }(t, x \Rightarrow t, x \Rightarrow t) \mid \\
& \text { true } \mid \text { false } \mid \text { if } t \text { then } t \text { else } t \mid \\
& \text { zero }|\operatorname{succ}(t)| \operatorname{rec}[x \Rightarrow \tau](t, t,(n, y) \Rightarrow t)|\operatorname{fix}[n \Rightarrow \tau]((n, y) \Rightarrow t)| \operatorname{match}(t, t, n \Rightarrow t) \mid \\
& \text { fold }[\tau](t) \mid \text { unfold } t \text { in } x \Rightarrow t \mid \text { unfold_pos } t \text { in } x \Rightarrow t \mid \\
& \Lambda \alpha . t|t[\tau]| \operatorname{err}[\tau] \mid \operatorname{ref}[[t, t]|\operatorname{let} x=t \operatorname{in} t| \operatorname{size}(t)
\end{aligned}
$$

Fig. 16. Grammar for annotated terms $t$, where $x, y$ and $n$ are term variables and $\alpha$ is a type variable.

termination arguments relies on a user-defined measure function. The fix term shown on the right corresponds to the desugaring of the recursive function on the left whose contracts are given by the require and ensuring keywords, and whose measure is given by the decreases keyword. The contract terms pre and post are such that $\mathrm{x}: \tau_{1} \vdash$ pre : Bool and $\mathrm{x}: \tau_{1}$, res : $\tau_{2} \vdash$ post : Bool, and the measure function measure satisfies $\mathrm{x}: \tau_{1} \vdash$ measure : Nat. The term pred is a function of type $\{n:$ Nat $\mid$ zero $<n\} \rightarrow$ Nat that returns the predecessor of numbers greater than zero.

We now explain how our type-checking algorithm ensures termination of such a function. Our type inference rule for fix is (Infer Fix). The side condition $n \notin f v(\operatorname{erase}(t))$ ensures that $n$ only appears in type annotations in $t$, and is not part of the computation. The other check corresponds to a proof by strong induction (over $n$ ) that the fix term has type $\forall n$ : Nat. $\tau$. Indeed, we have to check that $t$, the body of the fix term, has type $\tau$ (for some $n$ : Nat), under the assumption that $y$ (which is the variable representing the recursion) has type $\tau[n \mapsto m]$ for all $m<n$. The 'Unit $\rightarrow$ ' 


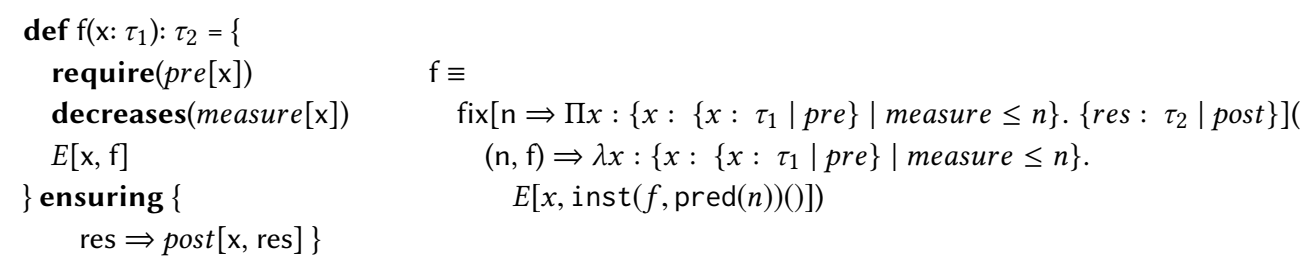

Fig. 17. Encoding named function with pre- and post-conditions are given by the require and ensuring, and measure given by the decreases keyword (left) into a terminating fixpoint recursion (right).

part of the type of $y$ corresponds to the fact that the operational semantics of fix replaces variable $y$ by the fix term under a lambda (as explained in Section 3).

The variable $p$ is a witness that the variable $y$ is equal to the fix term (under a lambda). This feature is useful for body-visible recursion, and is explained in Section 5.3.

Back to the encoding presented in Figure 17, we explain how the (Infer Fix) rule ensures that measure decreases at each recursive call of function $\mathrm{f}$. Assume that the premise of the (Infer Fix) rule holds, and that $\mathrm{f}$ is called with some value $v$ of type $\tau_{1}$, such that measure [ $v$ ] evaluates to some (term representing a) natural number $n$. By instantiating the premise of the (Infer Fix) rule for that particular $n$, we get that $E[x$, inst $(f, \operatorname{pred}(n))()]$ is well-typed under the condition that $f$ has type:

$\forall m:\{m:$ Nat $\mid m<n\}$. Unit $\rightarrow \Pi x:\left\{x:\left\{x: \tau_{1} \mid\right.\right.$ pre $\} \mid$ measure $\left.\leq m\right\} .\left\{\right.$ res $: \tau_{2} \mid$ post $\}$

First, in order for pred to be applied to $n$, we have to check that $n$ is non-zero, meaning that the measure of $v$ is strictly positive in the places where the recursive calls happen. This is ensured by the (Check Refinement) rule for checking refinement types (see Figure 15), which generates a verification condition.

Second, the rule (Infer Forall Instantiation) ensures that inst $(f, \operatorname{pred}(n))()$ takes arguments of type $\left\{x:\left\{x: \tau_{1} \mid\right.\right.$ pre $\} \mid$ measure $\left.\leq \operatorname{pred}(n)\right\}$. Therefore, if $f$ is applied recursively to an argument $v^{\prime}$, the rule (Check Refinement) ensures that measure $\left[v^{\prime}\right] \leq \operatorname{pred}(n)$ holds. Overall, we get measure $\left[v^{\prime}\right] \leq \operatorname{pred}(n)<n=$ measure[v], which ensures that the measures of arguments always decrease on recursive calls to $f$.

In our implementation, we do not go through the encoding with fix and forall types, but instead directly generate the verification conditions that correspond to the measure decreasing by using the left-hand-side form of Figure 17. Our system also supports mutually recursive functions (by requiring that the measure decreases for each call to a mutually recursive function), which can be encoded in the usual way by defining a fix term that returns a tuple of functions.

In the end, if the body of the function is well-typed, the (Infer Fix) rule infers the type:

$$
\forall n: \text { Nat. } \Pi x:\left\{x:\left\{x: \tau_{1} \mid \text { pre }\right\} \mid m \leq n\right\} .\left\{\text { res : } \tau_{2} \mid \text { post }\right\}
$$

One should note that this encoding imposes a scoping restriction on the original program, namely precondition, postcondition, and measure of a function $f$ cannot contain calls to $f$. This restriction has not proved limiting in our experience with benchmarks.

5.2.1 Lexicographic Orderings. Functions whose termination arguments require lexicographic orderings can be encoded by using two levels of recursions, which is a known technique that shows expressive power of System T [Girard 1990, Section 7.3.2]. We review how this encoding works in our system in the long version of our paper [Hamza et al. 2019] and show an example of Ackerman's function and its simple lexicographic measure. In our implementation, we support lexicographic measures directly. 


\subsection{Body-Visible Recursion}

In this section, we give more details about the (Infer Fix) and (Infer Rec) typing rules for recursion. They allow body-visible recursion which gives the type-checker access to the definition of a recursive function while type-checking the body of the recursive function itself.

The first thing to note is that we introduce an equality type containing the definition (in the type of $p$ ) in the context, while we do not know yet whether the body of the recursion is well-typed. Since our equality type is defined (in Section 4) for all terms, regardless of whether they are well-typed, this is perfectly legal. We show in the merge example of Figure 5 how body-visible recursion relieves the user from writing excessive specification annotations.

Assume we want to prove, on paper, that merge indeed returns a sorted list when given two sorted lists 11 and 12, by induction over size $(11)+\operatorname{size}(12)$. Consider the first branch of the if then else statement, where we return Cons $(x, \operatorname{merge}(x s, 12))$. By the induction hypothesis, we know that the recursive call merge $(x s, 12)$ is sorted, but this mere fact is not enough to conclude that Cons $(x, \operatorname{merge}(x s, 12))$ is sorted. By unfolding the definition of isSorted, we see that we need in addition to know that $x$ is smaller than the head of the result merge $(x s, 12)$.

Therefore, the property we prove by induction needs to be strengthened by saying that the head of the result, if non-empty, is equal to the (smallest) head of one the input lists. From that, we will know by the induction hypothesis that the head $h$ of merge (xs, 12) (if non-empty) is either the head of $x$ s or the head of 12. In the first case, we can deduce that $x$ is smaller than $h$ by using the fact that $11=\operatorname{Cons}(\mathrm{x}, \mathrm{xs})$ is sorted. In the second case, we have $h=\mathrm{y}$, and we know from the condition of the if then else statement that $x \leq y$. In both cases, we can conclude that the whole list Cons ( $x$, merge $(x s, 12))$ is sorted.

If we are to type-check the program above, and if we only know the return type of merge (xs , 12), that is $\{1$ : List | isSorted(1) \}, we will run into the same problem, and will not be able to conclude that Cons $(x, \operatorname{merge}(x s, 12))$ is sorted. In our type system, we get in addition access to the definition of merge while type-checking it, thanks to the $p$ variable of equality type in the (Infer Fix) rule. By unfolding the definition of $\operatorname{merge}(x s, 12)$, we conclude by case analysis that the head $h$ of merge (xs, 12) (if non-empty) is either the head of xs or the head of 12 (which is y).

Without body-visible recursion, the developer would need to strengthen the postcondition to:

$$
\begin{aligned}
\text { isEmpty(res) } \|(\text { ('isEmpty }(11) \& \& \text { head(res })==\text { head(I1)) } \\
\|(\text { !isEmpty(I2) \&\& head(res) }==\text { head }(I 2))
\end{aligned}
$$

In Inox, the external solver we use for verification conditions, definitions of recursive functions are unfolded automatically. Inox does incremental queries to SMT solvers. It first sends a query without unfolding at all, then a new query after unfolding once, and so on until a query succeeds or a timeout. Thanks to this approach, Inox does not rely on universal quantifiers to encode recursive functions. This feature is crucial to have such examples be verified without user intervention, and is here required to get the bodies of the calls to merge and isSorted when verifying the postcondition of merge.

\subsection{Unification in Type Inference}

In order to perform type inference, we expect a certain structure on the inferred types. For example, given term $t_{1} t_{2}$, we expect term $t_{1}$ to have a function type when inferring the type of an application. Furthermore, type inference must perform least upper bound computation for if, match and either_match terms, which adds more complexity to the system. We handle these considerations in a generalized manner by performing type unifications, defined in Figure 18 by using the type-level notations If Then Else, Let, Either_Match, and Match. 


$$
\begin{aligned}
& \text { unify }(E, \quad \text { Unit } \quad, \quad \text { Unit }) \triangleq \text { Unit } \\
& \text { unify }(E, \text { Bool }, \text { Bool }) \triangleq \text { Bool } \\
& \text { unify }(E, \quad \text { Nat , Nat }) \triangleq \text { Nat } \\
& \text { unify }(E, \quad T \quad, \quad T \quad) \triangleq T \\
& \operatorname{unify}(E, \quad \alpha \quad, \quad \alpha \quad) \triangleq \alpha \\
& \text { unify }\left(E, \quad \Pi x: A_{1} . B_{1} \quad, \quad \Pi x: A_{2} . B_{2}\right) \triangleq \Pi x: \text { unify }\left(E, A_{1}, A_{2}\right) \text {. unify }\left(E, B_{1}, B_{2}\right) \\
& \text { unify }\left(E, \quad \forall x: A_{1} . B_{1} \quad, \quad \forall x: A_{2} . B_{2}\right) \triangleq \forall x: \text { unify }\left(E, A_{1}, A_{2}\right) \text {. unify }\left(E, B_{1}, B_{2}\right) \\
& \text { unify }\left(E, \quad \sum x: A_{1} \cdot B_{1} \quad, \quad \sum x: A_{2} . B_{2}\right) \triangleq \Sigma x: \text { unify }\left(E, A_{1}, A_{2}\right) \text {. unify }\left(E, B_{1}, B_{2}\right) \\
& \text { unify }\left(E, \operatorname{Rec}\left(n_{1}\right)\left(\alpha \Rightarrow \tau_{1}\right), \operatorname{Rec}\left(n_{2}\right)\left(\alpha \Rightarrow \tau_{2}\right)\right) \triangleq \operatorname{Rec}\left(E\left[n_{1}, n_{2}\right]\right)\left(\alpha \Rightarrow \text { unify }\left(E, \tau_{1}, \tau_{2}\right)\right) \\
& \text { unify }\left(E, \quad\left\{x: A_{1} \mid p_{1}\right\},\left\{x: A_{2} \mid p_{2}\right\}\right) \triangleq\left\{x: \operatorname{unify}\left(E, A_{1}, A_{2}\right) \mid E\left[p_{1}, p_{2}\right]\right\} \\
& \text { unify }\left(E, \quad t_{1,1} \equiv t_{1,2} \quad, \quad t_{2,1} \equiv t_{2,2} \quad\right) \triangleq E\left[t_{1,1}, t_{2,1}\right] \equiv E\left[t_{1,2}, t_{2,2}\right] \\
& \text { If } \left.t_{1} \text { Then } \tau_{2} \text { Else } \tau_{3} \triangleq \text { unify(if } t_{1} \text { then _else _, } \tau_{2}, \tau_{3}\right) \\
& \text { Let } x=t \text { in } \tau \triangleq \text { unify(let } x=t \text { in , } \tau, \tau) \\
& \text { Either_Match } \left.\left(t, x \Rightarrow \tau_{1}, x \Rightarrow \tau_{2}\right) \triangleq \text { unify(either_match }\left(t, x \Rightarrow{ }_{-}, x \Rightarrow_{-}\right), \tau_{1}, \tau_{2}\right) \\
& \operatorname{Match}\left(t, \tau_{1}, n \Rightarrow \tau_{2}\right) \triangleq \operatorname{unify}\left(\operatorname{match}\left(t,{ }_{-}, n \Rightarrow_{-}\right), \tau_{1}, \tau_{2}\right)
\end{aligned}
$$

Fig. 18. Recursion schema for unification of two types, where $E$ is a context with two holes.

$$
\begin{gathered}
\frac{\Theta ; \Gamma \vdash t_{1} \Uparrow \operatorname{Rec}(\alpha \Rightarrow \tau) \quad \Theta ; \Gamma, x: \operatorname{Rec}(\alpha \Rightarrow \tau), p: t_{1} \equiv \operatorname{fold}(x) \vdash t_{2} \Uparrow \tau^{\prime} \quad \operatorname{spos}(\alpha, \tau)}{\Theta ; \Gamma \vdash \text { unfold } t_{1} \text { in } x \Rightarrow t_{2} \Uparrow \tau^{\prime}} \text { (Infer Unfold Gen) } \\
\frac{\Gamma \vdash t \Downarrow \tau[\alpha \mapsto \operatorname{Rec}(\alpha \Rightarrow \tau)] \quad \operatorname{spos}(\alpha, \tau)}{\Gamma \vdash \operatorname{fold}[\operatorname{Rec}(\alpha \Rightarrow \tau)](t) \Uparrow \operatorname{Rec}(\alpha \Rightarrow \tau)} \text { (Infer Fold Gen) }
\end{gathered}
$$

Fig. 19. Folding and unfolding without indices for strictly positive recursive types.

We draw attention here to the fact that our type checking and inference procedures are syntax directed and predictable, which enables a natural verification process through term-level hints. The algorithmic nature of our type checking precludes the verification of certain well-typed programs. However, our experience has shown that this limitation is largely inconsequential in practice and is outweighed by the predictable nature of the algorithm.

\subsection{Hiding Recursive Type Indices}

Beyond general rules of Figure 14, in Figure 19 we show two additional rules for 'fold' and 'unfold in' that ignore the indices hidden under the Rec type for strictly positive recursive types (which is the case for the List and Stream types). We write $\operatorname{spos}(\alpha, \tau)$ when a type variable appears only strictly positively in type $\tau$, meaning only to the right of $\Pi$ and $\forall$ types (see the long version of our paper [Hamza et al. 2019] for the precise definition). This enables us, under some conditions, to fold and unfold a strictly positive recursive type without worrying about indices.

Practically, given an element $l$ of type List[X] (resp. Stream[X]), we can unfold it (with the (Infer Unfold Gen) rule) to get its head and its tail of type List[X] (resp. Unit $\rightarrow$ Stream[X]). Conversely, we use the rule (Infer Fold Gen) to build a list or a stream from an element and a tail.

Strict positivity gives us the following key lemma that ensures the soundness of our rules with respect to our reducibility definition. This lemma states that when a type variable $\alpha$ appears only strictly positively in $\tau$, then quantifying with a forall type outside $\tau$ or inside a substitution for $\alpha$ is 
the same (as long as we are quantifying over a non-empty type $\tau_{1}$ ). This property is similar to the notions lim sup-pushable and lim inf-pullable [Abel 2008] and implies the soundness of our rules.

LemmA 5.2. Let $\tau$ and $\forall x: \tau_{1} . \tau_{2}$ be two types. Let $\alpha$ be a type variable that appears strictly positively in $\tau$. Let $\theta$ be a type interpretation such that $\llbracket \tau_{1} \rrbracket_{v}^{\theta}$ is not empty. We have:

$$
\llbracket \tau\left[\alpha \mapsto \forall x: \tau_{1} \cdot \tau_{2}\right] \rrbracket_{v}^{\theta}=\llbracket \forall x: \tau_{1} \cdot \tau\left[\alpha \mapsto \tau_{2}\right] \rrbracket_{v}^{\theta}
$$

\subsection{Type-Checking Algorithm Examples: Streams}

5.6.1 Constant Stream. The fix term and associated typing rules can also be used to express the kind of recursion used to define the streams in Section 2. We start by revisiting the constant stream, which in our notations can be written as an untyped term:

$$
\text { constant } \triangleq \mathrm{fix}(\text { constant } \Rightarrow \Lambda . \lambda x . \text { fold }(x, \lambda u \text {. constant }()[](x)))
$$

Assume we want to prove, on paper, that for any value $x$, constant []$(\mathrm{x})$ produces a non-diverging stream, i.e. a stream which is $n$-non-diverging for every $n \in \mathbb{N}$. A natural proof could be done by induction on $n$, as follows:

- $(n=0)$ constant[]( $\mathrm{x})$ is 0 -non-diverging, meaning that it reduces to a value of the form fold $(x, v)$ where $x$ and $v$ are values. This is clear from the code of constant, as this expression evaluates in a few steps to fold $\left(x, \lambda u\right.$. $\left(\lambda u^{\prime}\right.$. constant $\left.)()[](x)\right)$.

- $\left(n=n^{\prime}+1\right)$ Assume by induction that constant []$(\mathrm{x})$ is $n^{\prime}$-non-diverging. By definition of $n$-non-diverging, we get that $\operatorname{fold}(x, \lambda u$ : Unit. constant []$(x))$ is $n$-non-diverging. Since this term is equivalent to the term to which constant []$(x)$ evaluates, we conclude that constant []( $\mathrm{x})$ is $n$-non-diverging as well.

Our type system and type-checking algorithm can be used to simulate this proof by using an annotated version of constant:

$$
\text { constant } \triangleq \operatorname{fix}\left[n \Rightarrow \forall X: \text { Type. } X \rightarrow \operatorname{Stream}_{n}[X]\right]((n, \text { constant }) \Rightarrow \text { body }(n, \text { constant }))
$$

where body $(n$, constant) is a shorthand for

$$
\Lambda X . \lambda x: X . \text { fold }\left[\operatorname{Stream}_{n}[X]\right](x, \lambda u: \text { Unit. inst }(\text { constant, } \operatorname{pred}(n))()[X](x))
$$

By applying the (Infer Fix) rule presented above, we get the type

$$
\vdash \text { constant } \Uparrow \forall n: \text { Nat. } \forall X: \text { Type. } X \rightarrow \operatorname{Stream}_{n}[X] \text {. }
$$

The (Infer Fix) rule of our algorithm generates a check that corresponds to a (strong) induction that shows that for every $n \in \llbracket N a t \rrbracket_{\mathrm{V}}$, constant $[X](x)$ in $\llbracket S$ Steam $_{n}[X] \rrbracket_{\mathrm{v}}$ assuming that it in $\llbracket$ Stream $_{m}[X] \rrbracket_{\mathrm{v}}$ for all $m<n$ :

$$
\begin{aligned}
& n: \text { Nat, constant }: \forall m:\{m: \text { Nat } \mid m<n\} \text {. Unit } \rightarrow \forall X: \text { Type. } X \rightarrow \operatorname{Stream}_{n}[X] \vdash \\
& \operatorname{body}(\mathrm{n} \text {, constant }) \Downarrow \forall X: \text { Type. } X \rightarrow \operatorname{Stream}_{n}[X]
\end{aligned}
$$

After applying standard rules related to $\lambda$ and $\Lambda$, our algorithm will attempt to infer, using the (Infer Fold) rule, a type for the term:

$$
\text { fold } \left.\left[\operatorname{Stream}_{n}[X]\right](x, \lambda u \text { : Unit. inst(constant, } \operatorname{pred}(n))()[X](x)\right)
$$

In addition to the type-check that $n$ has type Nat, this rule generates two checks to cover the cases where $n$ is zero or non-zero. These correspond to the informal proof by induction given above for the non-divergence of constant. The first check reduces (after applying some straightforward rules) to checking that $\lambda u$ : Unit. inst(constant, $\operatorname{pred}(n))()[X](x)$ has type $\mathrm{T}$ (remember that basetype $_{\alpha}(X \times($ Unit $\left.\rightarrow \alpha))=X \times T\right)$, which goes through easily thanks to the rule (Check Top 1). 


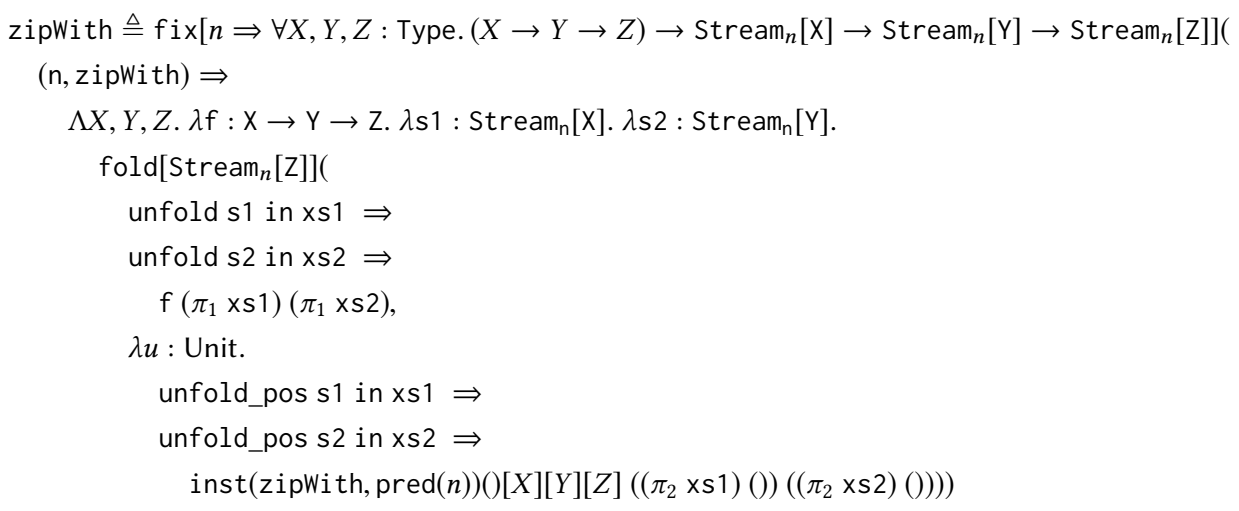

Fig. 20. An annotated term of our calculus to define zipWith.

The second check amounts to checking that inst(constant, pred $(n))()[\mathrm{X}](\mathrm{x})$ has type Stream $_{n^{\prime}}[X]$ under the assumption that $n \equiv \operatorname{succ}\left(n^{\prime}\right)$. By the context we know that inst (constant, $\operatorname{pred}(n))()[\mathrm{X}](\mathrm{x})$ has type $\operatorname{Stream} \operatorname{pred}(n)[X]$. Since $n^{\prime}$ and $\operatorname{pred}(n)$ are equivalent, we can use the rule (Check Recursive) (given in Figure 15) to convert between the two types.

Attempting to type-check the badConstant example from Section 2:

$$
\left.\mathrm{fix}\left[n \Rightarrow \forall X: \text { Type. } X \rightarrow \operatorname{Stream}_{n}[X]\right]((\mathrm{n} \text {, badConstant }) \Rightarrow \text { badBody(n, badConstant })\right)
$$

where badBody $(\mathrm{n}$, badConstant) stands for:

$\Lambda X . \lambda x: X$. fold $\left[\operatorname{Stream}_{n}[X]\right](x, \lambda u:$ Unit. inst(badConstant, $\left.\operatorname{pred}(n))()[X](x) . \operatorname{tail}()\right)$

will lead to an error in the second check (corresponding to the inductive case), since the extra call to tail() decreases the index of the stream by one.

5.6.2 ZipWith Function on Streams. We now revisit the zipWith function from Figure 7. We said in Section 2 that for every $n \in \mathbb{N}$, when s1 and s2 are $n$-non-diverging streams, then so is zipWith $(f, s 1, s 2)$. On paper, we can check by induction over $n \in \mathbb{N}$ that when s1 and s2 are $n$-non-diverging streams and $f$ is terminating, then zipWith $(f, \mathrm{~s} 1, \mathrm{~s} 2)$ (as written in Figure 7 , and ignoring type annotations and the erasable parameter for the moment) is also an $n$-non-diverging stream.

- $(n=0)$ It is indeed the case that we can access the head of zipWith ( $f, s 1, s 2)$ as long as we can access the heads $s 1$ and s2 (and as long as $f$ terminates).

- $\left(n=n^{\prime}+1\right)$ Let s1 and s2 be two $n$-non-diverging streams. By definition of non-diverging, we know that s1.tail() and s2.tail() are $n^{\prime}$-non-diverging. By induction hypothesis, zipWith(f,s1.tail(),s2.tail()) is $n^{\prime}$-non-diverging as well. This means that zipWith $(f, s 1, s 2)$ is $n$-non-diverging, which concludes the proof.

Accordingly, our type-checking algorithm is able to infer the type

$$
\forall n: \text { Nat. } \forall X, Y, Z \text { : Type. }(X \rightarrow Y \rightarrow Z) \rightarrow \operatorname{Stream}_{n}[\mathrm{X}] \rightarrow \operatorname{Stream}_{n}[\mathrm{Y}] \rightarrow \operatorname{Stream}_{n}[\mathrm{Z}]
$$

for the annotated zipWith term given in Figure 20. We use the term 'unfold in' to access the heads (with $\pi_{1}$ ). For the tails, we use instead 'unfold_pos in'. To understand the difference, we must first look at the (Infer Fold) rule for the fold term, which will generate two subgoals, one with $n=0$ and one with $n>0$. In the subgoal with $n=0$, we must check that the lambda term ' $\lambda u$ : Unit.[...]' has type T, which goes through directly thanks to (Check Top 1). Therefore, when 


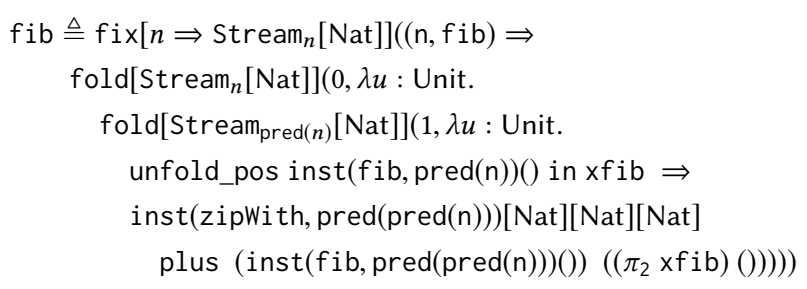

Fig. 21. An annotated term of our calculus to define the Fibonacci stream.

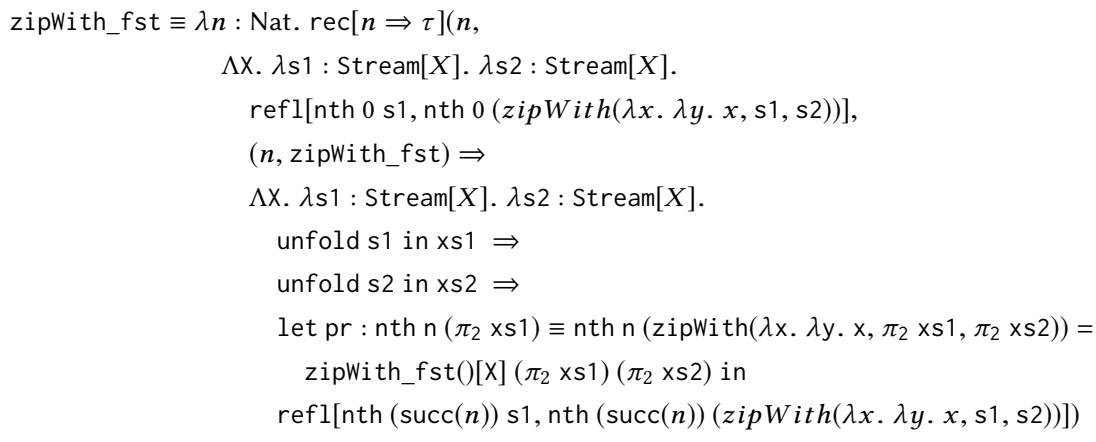

Fig. 22. An annotated term of our calculus to prove that zipping two streams with $\lambda x . \lambda y . x$ returns a stream equivalent to the first.

we are type-checking the body of the lambda, we know that $n>0$. We can thus access the tails using (Infer Unfold Positive), a variant of (Infer Unfold) that discards a subgoal with $n=0$ but requires proving $n>0$ instead.

5.6.3 Fibonacci Stream. We now consider the Fibonacci stream function from Figure 8. We can prove by induction that for all $n \in \mathbb{N}$, fib is an $n$-non-diverging stream. In our view, this corresponds to writing $\mathrm{fib}$ as in Figure 21.

When type-checking fib, we use the type of zipWith and instantiate it to pred(pred(n)), and get that: inst (zipWith, $\operatorname{pred}(\operatorname{pred}(n)))$ has type:

$\forall X, Y, Z:$ Type. $(X \rightarrow Y \rightarrow Z) \rightarrow \operatorname{Stream}_{\operatorname{pred}(\operatorname{pred}(n))}[\mathrm{X}] \rightarrow \operatorname{Stream}_{\operatorname{pred}(\operatorname{pred}(n))}[\mathrm{Y}] \rightarrow \operatorname{Stream}_{\operatorname{pred}(\operatorname{pred}(n))}[\mathrm{Z}]$

This is where we use the fact that zipWith returns an $n$-non-diverging stream when given n-non-diverging streams.

\subsection{Verification of Properties}

Finally, we show how to verify the property mentioned in Section 2: that zipping two streams s1 and $s 2$ with the function $\lambda x . \lambda y . x$ returns a stream equivalent to $s 1$.

We define a function zipWith_fst with the following type:

$\Pi n:$ Nat. $\forall \mathrm{X}:$ Type. $\Pi s 1$ : Stream $[\mathrm{X}] . \Pi s 2$ : Stream[X]. nth $n \mathrm{~s} 1 \equiv \mathrm{nth} n(z i p W i t h(\lambda x . \lambda y . x, \mathrm{~s} 1, \mathrm{~s} 2))$

For short, we denote this type as $\Pi n:$ Nat. $\tau$. The code of zipWith_fst is given in Figure 22 and makes use of the recursor rec. Our (Infer Rec) rule for inferring the type of rec is similar to fix. The difference is that rec allows references to $n$ in the code, so the computation is allowed to 


$$
\begin{aligned}
& \llbracket \perp \rrbracket_{\mathrm{v}}^{\theta} \triangleq\{\} \\
& \llbracket \tau_{1} \cap \tau_{2} \rrbracket_{\mathrm{v}}^{\theta} \triangleq \llbracket \tau_{1} \rrbracket_{\mathrm{v}}^{\theta} \cap \llbracket \tau_{2} \rrbracket_{\mathrm{v}}^{\theta} \quad \llbracket \tau_{1} \cup \tau_{2} \rrbracket_{\mathrm{v}}^{\theta} \triangleq \llbracket \tau_{1} \rrbracket_{\mathrm{v}}^{\theta} \cup \llbracket \tau_{2} \rrbracket_{\mathrm{v}}^{\theta} \\
& \llbracket \exists x: \tau_{1} \cdot \tau_{2} \rrbracket_{\mathrm{v}}^{\theta} \triangleq\left\{b \mid \exists a \in \llbracket \tau_{1} \rrbracket_{\mathrm{v}}^{\theta} \cdot b \in \llbracket \tau_{2}[x \mapsto a] \rrbracket_{\mathrm{v}}^{\theta}\right\} \quad \text { (existential) } \\
& \llbracket\left\{\left\{x: \tau_{1} \mid \tau_{2}\right\}\right\} \rrbracket_{\mathrm{v}}^{\theta} \triangleq\left\{a \in \llbracket \tau_{1} \rrbracket_{\mathrm{v}}^{\theta} \mid \exists b \in \llbracket \tau_{2}[x \mapsto a] \rrbracket_{\mathrm{v}}^{\theta}\right\} \quad \text { (refinement by type) } \\
& \llbracket \text { Let } x=t \text { in } \tau \rrbracket_{\mathrm{v}}^{\theta} \triangleq\left\{v \mid \exists a \in \text { Val. } t \hookrightarrow^{*} a \wedge v \in \llbracket \tau[x \mapsto a] \rrbracket_{\mathrm{v}}^{\theta}\right\}
\end{aligned}
$$

Fig. 23. Definition of reducibility for intersection, union, singleton types, and refinement by types.

depend on $n$. Moreover, rec does not use $\forall$ types. Finally, rec is analogous to a simple induction, while $f i x$ is analogous to a strong induction.

In the zipWith_fst example, applying this rule corresponds to a proof by induction over $n$, that for any two streams $s 1$ and $\mathrm{s} 2$, the $n$th element of $s 1$ is equal to the nth element of $z i p W i t h(\lambda x . \lambda y \cdot x, s 1, s 2)$.

In the base case, to type-check the term refl[nth 0 s1, nth $0(z i p W i t h(\lambda x . \lambda y . x, s 1, s 2))]$, we must make sure that the following equality holds: nth $0 \mathrm{~s} 1 \equiv \mathrm{nth} 0(\operatorname{zipWith}(\lambda x . \lambda y . x, \mathrm{~s} 1, \mathrm{~s} 2))$.

The corresponding type inference rule is (Infer Refl), which generates a verification condition.

In the inductive case, we explicitly instantiate our inductive hypothesis on the tails of s1 and s2 by using the let binding on variable pr. The equality given by the type of $\mathrm{pr}$ is then sufficient to prove what we wanted: $\mathrm{nth}(\operatorname{succ}(n)) \mathrm{s} 1 \equiv \mathrm{nth}(\operatorname{succ}(n))(z i p W i t h(\lambda x . \lambda y . x, \mathrm{~s} 1, \mathrm{~s} 2))$.

This example also illustrates that developers can express the desired versions of what others might call extensional equality (here: same results when calling $n t h)$ using $\Pi$ types and equi-reducibility $\equiv$; our type system does not impose any preferred form of extensional equality.

\section{FORMALIZATION IN THE COQ PROOF ASSISTANT}

We here give more details about our formalization of Theorem 5.1 in Coq 8.9.1 (including the rules from Section 5.5). Our proofs are available from

\section{https://github.com/epfl-lara/SystemFR/tree/oopsla2019}

We represent terms and types using a locally nameless representation [Charguéraud 2012], where free variables are named, and where local variables are bound using De Bruijn indices. Using this representation, lambdas and other binders can be seen as terms with holes, which can be filled with other terms (typically values). We use the Coq Equations library [Sozeau 2010] to define the reducibility logical relation $\llbracket \rrbracket_{\mathrm{v}}$. This library facilitates the use of functions which are defined recursively based on a well-founded measure.

We give an overview of our files (containing around 20k lines of code):

- Trees. $v$ contains the definitions of types and terms,

- Typing. $v$ gives all typing rules (containing rules from the paper and more),

- SmallStep. $v$ contains the operational semantics of the language,

- ReducibilityDefinition. $v$ contains the definition of reducibility,

- The Reducibility*.v files contain lemmas for the soundness of the rules from Typing. $v$,

- Reducibility. $v$ contains the proof that all typing rules from Typing. $v$ are sound with respect to the reducibility definition (which implies that Theorem 5.1 holds).

\subsection{Extensions of Formalization}

Our approach to formalization proved flexible and allowed us to extend the system with additional types. In addition to formalizing the types and rules for our type checking algorithm (figures 15 and 15), our Coq formalization also defines (Figure 23) reducibility for the bottom type, singleton types, 


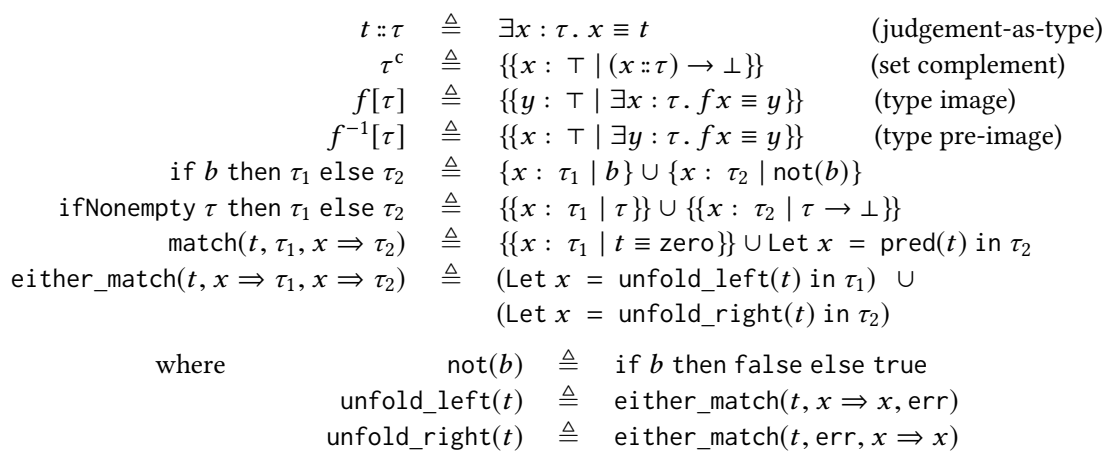

Fig. 24. Syntactic sugar for further expressive types

union and intersection types, and an existential type more abstract than dependent pair (and dual to $\left.\forall x: \tau_{1} . \tau_{2}\right)$. We also formalized refinement by type $\left\{\left\{x: \tau_{1} \mid \tau_{2}\right\}\right\}$, which generalizes refinements from computable terminating terms of our language to the condition of non-emptiness of arbitrary types in our system. Existential type and refinement by type can be viewed as second and first projections of dependent pairs. Together with existential and universal types, refinement by type allows us to refine types by quantified propositions, providing support for quantified preconditions and postconditions. Some of the type forms we present can be expressed using others; the set of type forms is not minimal.

We define further syntactic sugar, including precise if then else and match types (Figure 24), as an alternative to the If $t_{1}$ Then $t_{2}$ Else $t_{3}$ that relies on simplifications of Figure 18. We rely on Let type of Figure 23, and we use the ill-typed err term in the computations of (respectively) pred, unfold_left and unfold_right, to make sure that the denotations of the Let types are empty when $t$ does not reduce (respectively) to a strictly positive natural number, left $\left(t^{\prime}\right)$, or $\operatorname{right}\left(t^{\prime}\right)$ for some $t^{\prime}$. We proved soundness of typing rules that show that these types behave as expected. For instance, an if then else expression can be assigned an if then else type of the branches.

These types can be used to encode type level computation reminiscent to one present in Scala HList $^{3}$, as illustrated by the following example. Given a list $L$ : List [Bool] and types $\tau_{1}$ and $\tau_{2}$, we define a type TypeFromList $\left(L, \tau_{1}, \tau_{2}\right)$ that represents nested pairs from $\tau_{1}$ and $\tau_{2}$ according to the boolean values ( $\mathrm{fal}$ se for $\tau_{1}$ and true for $\tau_{2}$ ) and with nesting given by the length of $L$. For example, when $L$ is the list true, false, true then TypeFromList $(L, N a t, N a t \rightarrow N a t)$ represents nested tuples $w$ of the form $\left(a_{0},\left(a_{1},\left(a_{2}, \ldots\right)\right)\right)$ where $a_{0}:$ Nat, $a_{1}:$ Nat $\rightarrow$ Nat, $a_{2}:$ Nat.

TypeFromList $\left(L, \tau_{1}, \tau_{2}\right) \triangleq\left\{\left\{w: \top \mid \forall n:\{n:\right.\right.$ Nat $\mid n<\operatorname{size}(L)\} \cdot \operatorname{get}(w, n)::\left(\right.$ if $\operatorname{nth}(L, n)$ then $\tau_{1}$ else $\left.\left.\left.\tau_{2}\right)\right\}\right\}$

The function $n \operatorname{th}(L, n)$ returns the $n$th element of the list $L$ (defined as a recursive data type). The function get $(w, n)$ is also a term-level function that operates on nested pairs $w$ such that e.g. get $(w, 2)$ expands to $\pi_{1}\left(\pi_{2}\left(\pi_{2}(w)\right)\right)$. The type $x \equiv \operatorname{get}(w, n)$ in the expansion of refinement by judgement-as-type _::_(Figure 24 ) does not require type checking the get function; its semantics (see $\approx$ in Figure 13 ) is that get $(w, n)$ reduces to $x$ in the (untyped) operational semantics.

Furthermore, we prove soundness of multiple rules (such as congruence rules) for establishing the equality judgment $\Theta ; \Gamma \vdash t_{1} \equiv t_{2}$ described in Section 5 . We also prove soundness of a rule that unfolds the definition of a recursive function in the context, which is what is required by the solver in Section 5.3 to unfold the definition of merge. Such rules are a step towards justifying not only the verification condition generation but also verification condition solving.

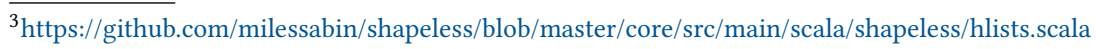




\section{IMPLEMENTATION AND EVALUATION}

We have implemented our bidirectional type checking procedure by writing an alternative verification-condition generator for Stainless ${ }^{4}$ [LARA 2019]. The code is merged into master and available in e.g. release version 0.4.0. Its functionality can be invoked with the--type-checker command line option in both scalac 2.12 and Dotty front end pipeline for Stainless. Thanks to these frontends, which provide a form of type inference, the type annotation burden is lessened for the user. Precise types such as indexed recursive types (which are not supported out of the box by these frontends) still need to be annotated manually.

The implementation was evaluated on benchmarks shown in Figure 25 totalling 14k LoC, collected from existing Stainless test suites and case studies. The proof/code ratio depends on the properties being proven. It can be 0 or close to 0 as in the merge example of Section 2, or higher than 1 when writing lemmas for proving detailed specifications (as in the Huffman coding example). The benchmarks reside in the frontends/benchmarks/typechecker/valid directory of Stainless. The streams benchmark relies on more expressive annotations of the Dotty compiler that we use to write recursive types, and is available in frontends/benchmarks/dotty-specific/typechecker. We use the following syntax to represent indexed types:

\begin{tabular}{|c|c|c|}
\hline & notation in this & encoding in Dotty front-end \\
\hline type & Stream $_{n}[X]$ & Stream $[X] @$ indexedAt(n) \\
\hline constructor & ${\text { fold }\left[\text { Stream }_{n}[X]\right]((h d, t l))}$ & indexedAt(n, Stream $[X](\mathrm{hd}, \mathrm{t} \mathrm{l}))$ \\
\hline
\end{tabular}

The suite of all benchmarks verifies in $\sim 6$ minutes in total when using implementation based on our type checker. The table shows the number of lines of codes within each benchmark, and the number of verification conditions which were generated by the type checker and verified by the SMT-backed Inox solver. The time given includes the time for generating the verification conditions and solving them, but not the parsing and compilation which is done by the Scala compiler scalac (which we use for some initial type inference and to obtain a tree representation of the program), nor the transformations which are internal to Stainless and which happen before using the type-checker To understand the impact of these time measurements, note that $11 \%$ of time of total verification time is spent in the bidirectional type-checking algorithm that generates verification conditions and that is the focus of this paper, $66 \%$ in checking the verification conditions using a SMT-backed solver Inox for recursive and higher-order functions, $13 \%$ for the parsing, name resolution and most of the type checking pipeline of Scala's scalac compiler, and $10 \%$ for extraction of scalac abstract syntax trees to Stainless trees. All times were measured on a Lenovo X1 Carbon laptop with an Intel i7-7500U and 16GB RAM.

To illustrate the diversity of benchmarks, we note that InsertionSort, QuickSorts, MergeSorts and StableSort feature various implementations of the sorting algorithms with the typical properties shown. ListMonad and OptionMonad show that the monadic laws hold for the List $[\mathrm{X}]$ and Option $[\mathrm{X}]$ types. The List and ListWithSize benchmarks feature a collection of common higherorder functions on lists such as map, filter, forall, etc., as well as many properties shown about the implementations. In the GodelNumbering benchmark, we prove that the pairing function $2^{x}(2 y+1)-1$ is a bijection between natural numbers and pairs of natural numbers using a series of lemmas about linear and non-linear arithmetic. Similar non-linearity is featured in the MoreExtendedEuclidGCD benchmark where we show that an implementation of the extended Euclid's algorithm indeed computes the greatest common divisor and the coefficients of Bézout's identity. Benchmarks also include persistent data structures [Okasaki 1998], ConcTree and ConcRope [Prokopec and Odersky 2015], explicit state model checker ReachabilityChecker, small interpreters, dynamic programming

$\overline{{ }^{4} \text { https://github.com/epfl-lara/stainless }}$ 


\begin{tabular}{|c|c|c|c|c|c|c|c|c|c|c|}
\hline Name & LoC & VCs & ime (s) & Name & LoC & VCs Time & Name & LoC & $\mathrm{VCs}^{\prime}$ & Time \\
\hline AbstractRefinementMap & 14 & 8 & 0.80 & FunSets & 134 & 191.11 & NatMonoidLaws & 75 & 39 & 0.71 \\
\hline Acc & 26 & 1 & 0.14 & GodelNumbering & 486 & 38984.00 & NaturalBuiltin & 25 & 8 & 0.11 \\
\hline Ackermann & 12 & 12 & 0.29 & HOInvocations & 19 & 20.07 & NestedLoop & 19 & 4 & 0.08 \\
\hline AliasPartial & 19 & 2 & 0.10 & HammingMemoized & 67 & $46 \quad 2.60$ & NotEquals & 22 & 1 & 0.09 \\
\hline AmortizedQueue & 155 & 64 & 3.92 & Heaps & 166 & $90 \quad 3.59$ & Numeric1 & 18 & 4 & 0.06 \\
\hline AnyDown & 24 & 3 & 0.21 & Huffman & 520 & $228 \quad 9.52$ & OddEven & 77 & 24 & 0.39 \\
\hline AssociativeFold & 102 & 36 & 2.14 & IgnoredField & 48 & 20.07 & OptionMonad & 47 & 9 & 0.26 \\
\hline AssociativeList & 66 & 28 & 0.80 & IndirectHO & 19 & 20.04 & Overrides & 25 & 9 & 0.24 \\
\hline BalancedParentheses & 410 & 214 & 25.76 & Induction & 415 & $150 \quad 3.04$ & PackratParsing & 137 & 31 & 1.06 \\
\hline BasicReal & 28 & 6 & 0.18 & InsertionSort & 85 & 310.46 & ParBalance & 250 & 88 & 1.60 \\
\hline Basics & 442 & 137 & 3.60 & IntSet & 65 & $29 \quad 0.51$ & PartialCompiler & 66 & 38 & 10.67 \\
\hline BestRealTypes & 27 & 1 & 0.14 & IntSetProp & 66 & $21 \quad 0.57$ & PartialKVTrace & 77 & 19 & 3.69 \\
\hline BigIntMonoidLaws & 38 & 9 & 1.96 & IntSetUnit & 60 & $23 \quad 0.51$ & Patterns & 28 & 10 & 0.78 \\
\hline BinarySearch & 18 & 7 & 6.61 & Justify & 79 & $46 \quad 0.90$ & Peano & 36 & 12 & 0.28 \\
\hline BinomialHeap & 189 & 22 & 0.95 & Knapsack & 73 & $23 \quad 0.30$ & PositiveMap & 43 & 15 & 0.41 \\
\hline BitsTricks & 103 & 35 & 0.72 & LazyNumericalRep & 158 & $29 \quad 1.15$ & PreInSpecs & 27 & 6 & 0.09 \\
\hline BooleanOps & 50 & 12 & 0.41 & LazySelectionSort & 69 & 210.40 & PropositionalLogic & 89 & 114 & 16.04 \\
\hline BottomUpMergeSort & 127 & 62 & 2.80 & LeftPad & 65 & $25 \quad 1.10$ & Queue & 28 & 9 & 0.25 \\
\hline Bytes & 33 & 7 & 0.12 & List & 1006 & 52916.07 & QuickSorts & 220 & 131 & 5.65 \\
\hline ChurchNum & 21 & 0 & 0.03 & ListMonad & 86 & $29 \quad 0.97$ & ReachabilityChecker & 561 & 282 & 15.34 \\
\hline Client & 20 & 1 & 0.09 & ListMonoidLaws & 47 & $22 \quad 0.46$ & RealTimeQueue & 77 & 9 & 0.33 \\
\hline ConcRope & 493 & 254 & 49.21 & ListWithSize & 169 & $105 \quad 1.56$ & RedBlackTree & 115 & 49 & 1.52 \\
\hline ConcTree & 335 & 180 & 9.44 & LiteralMaps & 25 & $\begin{array}{ll}0 & 0.05\end{array}$ & SearchLinkedList & 59 & 22 & 0.27 \\
\hline CountTowardsZero & 16 & 7 & 0.08 & Longs & 36 & $\begin{array}{ll}8 & 3.53\end{array}$ & Shorts & 33 & 7 & 0.10 \\
\hline Countable & 29 & 17 & 0.49 & Map & 21 & $3 \quad 0.11$ & SimpInterpret & 71 & 18 & 1.18 \\
\hline Deque & 241 & 80 & 3.73 & MapGetOrElse2 & 19 & 20.14 & StableSorter & 129 & 52 & 1.51 \\
\hline DivisionByZero & 21 & 3 & 0.06 & MapGetPlus & 20 & $10 \quad 0.41$ & Streams & 87 & 147 & 4.41 \\
\hline EffectfulPost & 18 & 1 & 0.08 & McCarthy 91 & 24 & $\begin{array}{ll}8 & 0.17\end{array}$ & Termination_passing1 & 37 & 10 & 0.16 \\
\hline EitherLaws & 34 & 5 & 0.15 & Mean & 13 & $2 \quad 0.27$ & Theorem & 28 & 1 & 0.05 \\
\hline Factorial & 47 & 23 & 0.60 & MergeSorts & 301 & $167 \quad 4.51$ & ToChurch & 27 & 4 & 0.07 \\
\hline Fibonacci & 19 & 7 & 0.10 & Methods & 32 & 130.28 & Trees1 & 29 & 11 & 0.43 \\
\hline FiniteSort & 61 & 14 & 1.02 & MicroTests & 2246 & 50515.59 & TweetSet & 494 & 253 & 11.91 \\
\hline FiniteStreams & 42 & 13 & 0.30 & Monoid & 37 & $12 \quad 0.49$ & UpDown & 49 & 11 & 0.25 \\
\hline FlatMap & 60 & 20 & 0.66 & MoreExtendedEuclidGCD & 74 & 397.18 & Viterbi & 120 & 19 & 0.33 \\
\hline Foldr & 19 & 3 & 0.09 & MySet & 18 & 20.03 & XPlus2N & 19 & 4 & 0.07 \\
\hline FoolProofAdder & 13 & 1 & 0.04 & NNF & 135 & $177 \quad 17.77$ & example & 95 & 70 & 1.74 \\
\hline Formulas & 55 & 30 & 2.30 & NNFSimple & 107 & $103 \quad 5.27$ & recfun & 66 & 38 & 0.89 \\
\hline
\end{tabular}

Fig. 25. Summary of evaluation results for our verification tool, featuring lines of code, number of verification conditions (VCs), and type-checking times (including generation of VCs and checking of VCs by Inox).

algorithms such as Viterbi and Knapsack, and benchmarks solving assignments from Scala MOOCs (see https://courseware.epfl.ch/ or https://www.coursera.org/specializations/scala).

\section{RELATED WORK}

In this paper, we tackle program verification using a type-theoretic approach. Other techniques, such as symbolic execution for verification (see e.g. [Hallahan et al. 2019; Nguyen et al. 2017]) or using term rewriting systems (see e.g. [Giesl et al. 2011, 2006, 2004]) can be used instead or in complement to our approach. The use of type checking in verification appears in program verifiers such as F* [Ahman et al. 2017; Swamy et al. 2016, 2013] and Liquid Haskell [Vazou et al. 2013, 2014, 2018]. Like Dafny [Leino 2010] and Stainless, these systems rely on SMT solvers to automatically discharge verification conditions.

$\mathrm{F}^{*}$ is a dependently-typed programming language which supports a rich set of effects (such as divergence, mutation, etc.). To the best of our knowledge $\mathrm{F}^{*}$ does not support proving termination of functions operating on infinite data structures such as streams. Stainless has a desugaring pass that handles local state and certain forms of unique references. Furthermore, for semantic modeling of global state, a user can use monad syntax (Scala's for comprehensions) explicitly in the surface language, which Scala compiler desugars into higher-order functions that our system can handle. Overall, $\mathrm{F}^{*}$ supports a more expressive class of effects than what Stainless currently handles. The goal of Liquid Haskell [Vazou et al. 2018] is to add refinement types to Haskell, a callby-need language (while we focus here on call-by-value languages). The system supports a sound 
verification procedure which relies on decidable theories of SMT. The supported language imposes certain restrictions on refinement occurrences and the metatheory does not feature recursive types (although they are supported by the implementation). Similarly to our body-visible recursion, Liquid Haskell allows the type checker to access the body of recursive functions (for recursive calls) while type-checking the function itself using a technique called refinement reflection [Vazou et al 2018]. Dafny verifier [Leino 2010] supports imperative and object-oriented as well as functional programming, including inductive and coinductive types, recursion using ordinals, quantifiers. We are not aware of metatheory to justify the soundness of Dafny. In contrast, we provide a mechanized proof of soundness for System FR, which increases confidence in VC generation soundness. This is important because it is easy to construct paradoxes when combining features such as impredicativity and contravariant recursion. Being based on a reducibility relation, our proof can be used as a basis to add new language features in a compositional way. Several other systems have been recently formalized, such as MetaCoq [Sozeau et al. 2019].

Proof assistants with great expressive power include Isabelle [Blanchette et al. 2017; Nipkow et al. 2002b], Coq [Barras 2010; Bertot and Castéran 2004b; Sacchini 2013], Idris [Brady 2013], Agda [Abel 2010; Norell 2007], $\mathrm{PML}_{2}$ [Lepigre 2017], Lean [de Moura 2016] and Zombie [Casinghino et al. 2014]. When coinduction is supported in these systems (which is not always the case), it is typically through special constructs for coinductive types and their corresponding cofixpoint operators, dual to inductive types and their fixpoint operators. In System FR, we instead treat induction and coinduction in a uniform way (see e.g. Section 5.6). Our type system features a single kind of recursive types which allow uniform definition of inductive, coinductive and mixed recursive types. Our operational semantics further rely on the fix operator which roughly corresponds to general recursion in mainstream programming call-by-value languages. An alternative approach to uniform handling of recursion and corecursion is given in [Gianantonio and Miculan 2002]. We were able to encode the first example from this work (in our Streams benchmark) which generates a stream and whose termination proof requires a combination of inductive and coinductive reasoning. Using our system, we expressed that using a simple lexicographic measure combining the index of the type of the produced stream with the inductive measure. In a private communication, Andreas Abel showed us that Agda can also handle this example.

Our system follows Nuprl [Constable et al. 1986] (and other computational type theories) style of starting out with an untyped calculus and then introducing various types to classify untyped terms based on their behaviors. Nuprl supports a very expressive type system, which covers the types we present in this paper, except impredicative polymorphism. In a large development, Nuprl's metatheory has been formalized in Coq [Anand and Rahli 2014]. One part of that formalization relies on the use of the Coq axiom of functional choice FunctionalChoice_on, which gives a function $f$ from $A$ to $B$ when a formula of the form $\forall a: A$. $\exists b: B . \phi(a, b)$ holds. Nuprl does not use SMT solvers for automation, but relies instead on built-in and user-defined tactics similarly to Coq

Our reducibility definition for recursive type is inspired from step-indexed logical relations [Ahmed 2006]. The main difference is that the indices in step-indexed logical relations do not appear at the level of types, but at the level of the logical relation that gives meaning to types. In System FR we internalize the indices at the level of recursive types in order to give more expressive power to the users, and let them specify decreasing measures for recursive functions that manipulate infinite data structures. This treatment of recursive types is similar to the TORES [JacobRao et al. 2018] type system, where recursive types can be indexed by an arbitrary index language. We only support recursive types indexed by natural numbers. TORES provides a decision procedure for a rich type system with inductive types, coinductive types, and indexed recursive types, yet its metatheory does not handle polymorphism nor refinement types. 
Our termination criterion (rule for fix) is inspired by type-based termination [Abel 2004, 2007, 2008, 2012; Abel and Pientka 2013; Barthe et al. 2004]. Such work also typically uses two different kinds of recursion, one for induction and one for coinduction. In type-based termination, instead of requiring that a measure on the arguments of recursive calls decreases, we require that recursive functions are called at a type which is strictly smaller than the type of the caller. Our fix operator produces a term with a forall type, which is similar to the implicit function type of the implicit calculus of constructions [Miquel 2001]. Our termination measure on types can then be understood as a measure on the implicit argument of a function with an implicit function type. Earlier work on type-based termination checking includes simpler type systems such as DML [Xi 2001].

A proof of concept of extension of Dotty compiler by a restricted set of (mostly numerical) refinement predicates was presented in [Schmid and Kunčak 2016], without soundness proof. Developments specific to Scala include dependent object types [Amin 2016] which focus on path dependency instead of predicate refinement and, by design, admit possibly non-terminating programs. System FR does not attempt to support records, let alone path dependency. Stainless relies on Scala compiler front ends to obtain a symbol-resolved syntax tree. For example, implicit parameters in Scala [Odersky et al. 2018] become ordinary explicit parameters by the time Stainless processes them. Conversely, many of the types we define in System FR do not have their counterparts in Scala. An upcoming PhD thesis of Nicolas Voirol [Voirol 2019] presents a system related to System FR that more closely follows the expressive power of Inox solver on which Stainless relies.

\section{CONCLUSION}

We have presented System FR, a formalized type system and a bidirectional type checking algorithm that can serve as a basis of a verifier for higher-order functional programs. We were able to verify correctness and safety of a wide range of benchmarks amounting to $14 \mathrm{k}$ lines of code and proofs. Our formalization suggests that lazy data structures and non-covariant recursion are tractable and that explicit indices required in the general framework can be eliminated in commonly occurring cases. Our type system incorporates $\Pi$ and $\Sigma$ types, yet it also supports their variants $(\forall$ and $\exists$ ) that correspond to infinite intersections and unions. Along with support for singleton types and refinements, we obtained a rich framework to approximate program semantics, which can also help further type, invariant, and measure inference algorithms. Our experience confirms an advantage of the semantic-based soundness proof: once we adopt an approach of interpreting types as sets of terms, we are less dependent on a particular choice of syntactic rules; we can introduce new classes of types and new rules for verification condition generation and solving, as long as we can justify them semantically. When viewing types in our system as propositions, we obtain an expressive quantified logic. We have proven the soundness of this logic in Coq using countable interpretation of ground System FR types. The success in verification of our benchmarks suggests that the rules of System FR work well for many properties of functional programs. Furthermore, refinement by type along with intersections and unions (Section 6.1) allows System FR to describe types whose interpretations are undecidable countable sets from higher levels of arithmetical hierarchy.

\section{ACKNOWLEDGMENTS}

Work supported in part by Swiss National Science Foundation project 200021_175676 "Scaling Predicate Types”. We thank Ravichandhran Kandhadai Madhavan for numerous discussions about termination for higher-order functional programs; Romain Ruetschi for his valuable contributions to Stainless implementation; Paolo G. Giarrusso for interesting discussions and very relevant pointers on type theory; Andreas Abel for explaining us how to mix induction and co-induction in Agda. Thanks to Yoan Géran who implemented another prototype of our algorithm, found formalization discrepancies and proposed improvements to the typing rules. 


\section{REFERENCES}

Andreas Abel. 2004. Termination checking with types. ITA 38, 4 (2004), 277-319. https://doi.org/10.1051/ita:2004015

Andreas Abel. 2007. Type-based termination: a polymorphic lambda-calculus with sized higher-order types. Ph.D. Dissertation. Ludwig Maximilians University Munich. http://d-nb.info/984765581

Andreas Abel. 2008. Semi-Continuous Sized Types and Termination. Logical Methods in Computer Science 4, 2 (2008). https://doi.org/10.2168/LMCS-4(2:3)2008

Andreas Abel. 2010. MiniAgda: Integrating Sized and Dependent Types. In Partiality and Recursion in Interactive Theorem Provers, PAR@ITP 2010, Edinburgh, UK, July 15, 2010 (EPiC Series), Ekaterina Komendantskaya, Ana Bove, and Milad Niqui (Eds.), Vol. 5. EasyChair, 18-32. https://doi.org/10.29007/322q

Andreas Abel. 2012. Type-Based Termination, Inflationary Fixed-Points, and Mixed Inductive-Coinductive Types. In Proceedings 8th Workshop on Fixed Points in Computer Science, FICS 2012, Tallinn, Estonia, 24th March 2012. (EPTCS), Dale Miller and Zoltán Ésik (Eds.), Vol. 77. 1-11. https://doi.org/10.4204/EPTCS.77.1

Andreas Abel and Brigitte Pientka. 2013. Wellfounded recursion with copatterns: a unified approach to termination and productivity. In ACM SIGPLAN International Conference on Functional Programming, ICFP'13, Boston, MA, USA - September 25 - 27, 2013, Greg Morrisett and Tarmo Uustalu (Eds.). ACM, 185-196. https://doi.org/10.1145/2500365.2500591

Danel Ahman, Catalin Hritcu, Kenji Maillard, Guido Martínez, Gordon D. Plotkin, Jonathan Protzenko, Aseem Rastogi, and Nikhil Swamy. 2017. Dijkstra monads for free. In Proceedings of the 44th ACM SIGPLAN Symposium on Principles of Programming Languages, POPL 2017, Paris, France, fanuary 18-20, 2017, Giuseppe Castagna and Andrew D. Gordon (Eds.). ACM, 515-529. http://dl.acm.org/citation.cfm?id=3009878

Amal J. Ahmed. 2006. Step-Indexed Syntactic Logical Relations for Recursive and Quantified Types. In Programming Languages and Systems, 15th European Symposium on Programming, ESOP 2006, Held as Part of the foint European Conferences on Theory and Practice of Software, ETAPS 2006, Vienna, Austria, March 27-28, 2006, Proceedings (Lecture Notes in Computer Science), Peter Sestoft (Ed.), Vol. 3924. Springer, 69-83. https://doi.org/10.1007/11693024_6

Nada Amin. 2016. Dependent Object Types. Ph.D. Dissertation. EPFL. https://doi.org/10.5075/epfl-thesis-7156

Abhishek Anand and Vincent Rahli. 2014. Towards a Formally Verified Proof Assistant. In Interactive Theorem Proving, Gerwin Klein and Ruben Gamboa (Eds.). Springer International Publishing, Cham, 27-44. https://doi.org/10.1007/978-3319-08970-6_3

Mike Barnett, K. Rustan M. Leino, and Wolfram Schulte. 2004. The Spec\# Programming System: An Overview. In Construction and Analysis of Safe, Secure, and Interoperable Smart Devices, International Workshop, CASSIS 2004, Marseille, France, March 10-14, 2004, Revised Selected Papers (Lecture Notes in Computer Science), Gilles Barthe, Lilian Burdy, Marieke Huisman, Jean-Louis Lanet, and Traian Muntean (Eds.), Vol. 3362. Springer, 49-69. https://doi.org/10.1007/978-3-540-30569-9_3

Bruno Barras. 2010. Sets in Coq, Coq in Sets. F. Formalized Reasoning 3, 1 (2010), 29-48. https://doi.org/10.6092/issn.19725787/1695

Gilles Barthe, Maria João Frade, Eduardo Giménez, Luís Pinto, and Tarmo Uustalu. 2004. Type-based termination of recursive definitions. Mathematical Structures in Computer Science 14, 1 (2004), 97-141. https://doi.org/10.1017/S0960129503004122

Gilles Barthe, Benjamin Grégoire, and Colin Riba. 2008. A Tutorial on Type-Based Termination. In Language Engineering and Rigorous Software Development, International LerNet ALFA Summer School 2008, Piriapolis, Uruguay, February 24 March 1, 2008, Revised Tutorial Lectures. 100-152. https://doi.org/10.1007/978-3-642-03153-3_3

Yves Bertot and Pierre Castéran. 2004a. Interactive Theorem Proving and Program Development - Coq'Art: The Calculus of Inductive Constructions. Springer. https://doi.org/10.1007/978-3-662-07964-5

Yves Bertot and Pierre Castéran. 2004b. Interactive Theorem Proving and Program Development - Coq'Art: The Calculus of Inductive Constructions. Springer. https://doi.org/10.1007/978-3-662-07964-5

Régis Blanc and Viktor Kuncak. 2015. Sound reasoning about integral data types with a reusable SMT solver interface. In Proceedings of the 6th ACM SIGPLAN Symposium on Scala, Scala@PLDI 2015, Portland, OR, USA, June 15-17, 2015. 35-40. https://doi.org/10.1145/2774975.2774980

Jasmin Christian Blanchette, Aymeric Bouzy, Andreas Lochbihler, Andrei Popescu, and Dmitriy Traytel. 2017. Friends with Benefits - Implementing Corecursion in Foundational Proof Assistants. In Programming Languages and Systems - 26th European Symposium on Programming, ESOP 2017, Held as Part of the European foint Conferences on Theory and Practice of Software, ETAPS 2017, Uppsala, Sweden, April 22-29, 2017, Proceedings (Lecture Notes in Computer Science), Hongseok Yang (Ed.), Vol. 10201. Springer, 111-140. https://doi.org/10.1007/978-3-662-54434-1_5

Edwin Brady. 2013. Idris: general purpose programming with dependent types. In Proceedings of the 7th Workshop on Programming languages meets program verification, PLPV 2013, Rome, Italy, fanuary 22, 2013, Matthew Might, David Van Horn, Andreas Abel, and Tim Sheard (Eds.). ACM, 1-2. https://doi.org/10.1145/2428116.2428118

Chris Casinghino, Vilhelm Sjöberg, and Stephanie Weirich. 2014. Combining proofs and programs in a dependently typed language. In The 41st Annual ACM SIGPLAN-SIGACT Symposium on Principles of Programming Languages, POPL '14, San Diego, CA, USA, January 20-21, 2014, Suresh Jagannathan and Peter Sewell (Eds.). ACM, 33-46. https://doi.org/10.1145/ 2535838.2535883

Proc. ACM Program. Lang., Vol. 3, No. OOPSLA, Article 166. Publication date: October 2019. 
Arthur Charguéraud. 2012. The Locally Nameless Representation. F. Autom. Reasoning 49, 3 (2012), 363-408. https: //doi.org/10.1007/s10817-011-9225-2

Robert L. Constable, Stuart F. Allen, Mark Bromley, Rance Cleaveland, J. F. Cremer, R. W. Harper, Douglas J. Howe, Todd B. Knoblock, N. P. Mendler, Prakash Panangaden, James T. Sasaki, and Scott F. Smith. 1986. Implementing mathematics with the Nuprl proof development system. Prentice Hall. http://dl.acm.org/citation.cfm?id=10510

Leonardo de Moura. 2016. Formalizing Mathematics using the Lean Theorem Prover. In International Symposium on Artificial Intelligence and Mathematics, ISAIM 2016, Fort Lauderdale, Florida, USA, Fanuary 4-6, 2016. http://isaim2016.cs.virginia. edu/papers/ISAIM2016_Proofs_DeMoura.pdf

David L. Detlefs, K. Rustan M. Leino, Greg Nelson, and James B. Saxe. 1998. Extended Static Checking. Technical Report 159. COMPAQ Systems Research Center.

Pietro Di Gianantonio and Marino Miculan. 2002. A Unifying Approach to Recursive and Co-recursive Definitions. In Types for Proofs and Programs, Second International Workshop, TYPES 2002, Berg en Dal, The Netherlands, April 24-28, 2002, Selected Papers (Lecture Notes in Computer Science), Herman Geuvers and Freek Wiedijk (Eds.), Vol. 2646. Springer, 148-161. https://doi.org/10.1007/3-540-39185-1_9

Jürgen Giesl, Matthias Raffelsieper, Peter Schneider-Kamp, Stephan Swiderski, and René Thiemann. 2011. Automated Termination Proofs for Haskell by Term Rewriting. ACM Trans. Program. Lang. Syst. 33, 2 (Feb. 2011), 7:1-7:39. https: //doi.org/10.1145/1890028.1890030

J Giesl, S Swiderski, P Schneider-Kamp, and R Thiemann. 2006. Automated termination analysis for Haskell: From term rewriting to programming languages. In Rewriting techniques and Applications, Vol. 4098. 297-312.

Jürgen Giesl, René Thiemann, Peter Schneider-Kamp, and Stephan Falke. 2004. Automated Termination Proofs with AProVE. In Rewriting Techniques and Applications, 15th International Conference, RTA 2004, Aachen, Germany, fune 3-5, 2004, Proceedings. 210-220. https://doi.org/10.1007/978-3-540-25979-4_15

Jean-Yves Girard. 1971. Une Extension De L'Interpretation De Gödel a L'Analyse, Et Son Application a L'Elimination Des Coupures Dans L'Analyse Et La Theorie Des Types. Studies in Logic and the Foundations of Mathematics 63 (1971), 63-92.

Jean-Yves Girard. 1990. Proofs and types. Cambridge University Press. http://www.paultaylor.eu/stable/prot.pdf

M. J. C. Gordon and T. F. Melham. 1993. Introduction to HOL, a theorem proving environment for higher-order logic. Cambridge University Press, Cambridge, England.

William T. Hallahan, Anton Xue, Maxwell Troy Bland, Ranjit Jhala, and Ruzica Piskac. 2019. Lazy counterfactual symbolic execution. In Proceedings of the 40th ACM SIGPLAN Conference on Programming Language Design and Implementation, PLDI 2019, Phoenix, AZ, USA, June 22-26, 2019., Kathryn S. McKinley and Kathleen Fisher (Eds.). ACM, 411-424. https: //doi.org/10.1145/3314221.3314618

Jad Hamza, Nicolas Voirol, and Viktor Kunčak. 2019. System FR as Foundations for Stainless. arXiv:cs.LO/1904.03482

Robert Harper. 2016. Practical foundations for programming languages. Cambridge University Press.

John Harrison. 2009. Handbook of Practical Logic and Automated Reasoning. Cambridge University Press.

John Harrison. 2017. HOL Light Tutorial. https://www.cl.cam.ac.uk/ jrh13/hol-light/tutorial.pdf. Retrieved 19 July 2019.

Lars Hupel and Viktor Kuncak. 2016. Translating Scala Programs to Isabelle/HOL (System Description). In International foint Conference on Automated Reasoning (IFCAR).

Rohan Jacob-Rao, Brigitte Pientka, and David Thibodeau. 2018. Index-Stratified Types. In 3rd International Conference on Formal Structures for Computation and Deduction, FSCD 2018, July 9-12, 2018, Oxford, UK (LIPIcs), Hélène Kirchner (Ed.), Vol. 108. Schloss Dagstuhl - Leibniz-Zentrum fuer Informatik, 19:1-19:17. https://doi.org/10.4230/LIPIcs.FSCD.2018.19

EPFL IC LARA. 2019. Stainles: Formal Verification for Scala. http://stainless.epfl.ch/.

K Rustan M Leino. 2010. Dafny: An Automatic Program Verifier for Functional Correctness. In Logic for Programming, Artificial Intelligence, and Reasoning. 348-370. https://doi.org/10.1007/978-3-642-17511-4\{_\}20

Rodolphe Lepigre. 2017. Semantics and Implementation of an Extension of ML for Proving Programs. (Sémantique et Implantation d'une Extension de ML pour la Preuve de Programmes). Ph.D. Dissertation. Grenoble Alpes University, France. https: //tel.archives-ouvertes.fr/tel-01590363

Alexandre Miquel. 2001. The Implicit Calculus of Constructions. In TLCA. 344-359. https://doi.org/10.1007/3-540-45413-6_27

Phuc C. Nguyen, Sam Tobin-Hochstadt, and David Van Horn. 2017. Higher order symbolic execution for contract verification and refutation. F. Funct. Program. 27 (2017), e3. https://doi.org/10.1017/S0956796816000216

Tobias Nipkow, Lawrence C. Paulson, and Markus Wenzel. 2002a. Isabelle/HOL - A Proof Assistant for Higher-Order Logic. Lecture Notes in Computer Science, Vol. 2283. Springer. https://doi.org/10.1007/3-540-45949-9

Tobias Nipkow, Lawrence C. Paulson, and Markus Wenzel. 2002b. Isabelle/HOL - A Proof Assistant for Higher-Order Logic. Lecture Notes in Computer Science, Vol. 2283. Springer. https://doi.org/10.1007/3-540-45949-9

Ulf Norell. 2007. Towards a practical programming language based on dependent type theory. Ph.D. Dissertation. Department of Computer Science and Engineering, Chalmers University of Technology, SE-412 96 Göteborg, Sweden.

Martin Odersky, Olivier Blanvillain, Fengyun Liu, Aggelos Biboudis, Heather Miller, and Sandro Stucki. 2018. Simplicitly: foundations and applications of implicit function types. PACMPL 2, POPL (2018), 42:1-42:29. 
Martin Odersky, Lex Spoon, and Bill Venners. 2008. Programming in Scala: a comprehensive step-by-step guide. Artima Press. Chris Okasaki. 1998. Purely Functional Data Structures. Cambridge University Press.

Aleksandar Prokopec and Martin Odersky. 2015. Conc-Trees for Functional and Parallel Programming. In Languages and Compilers for Parallel Computing, LCPC. 254-268. https://doi.org/10.1007/978-3-319-29778-1_16

Jorge Luis Sacchini. 2013. Type-Based Productivity of Stream Definitions in the Calculus of Constructions. In 28th Annual ACM/IEEE Symposium on Logic in Computer Science, LICS 2013, New Orleans, LA, USA, June 25-28, 2013. IEEE Computer Society, 233-242. https://doi.org/10.1109/LICS.2013.29

Georg Stefan Schmid and Viktor Kunčak. 2016. SMT-Based Checking of Predicate-Qualified Types for Scala. In Scala Symposium.

Matthieu Sozeau. 2010. Equations: A Dependent Pattern-Matching Compiler. In Interactive Theorem Proving, First International Conference, ITP 2010, Edinburgh, UK, July 11-14, 2010. Proceedings (Lecture Notes in Computer Science), Matt Kaufmann and Lawrence C. Paulson (Eds.), Vol. 6172. Springer, 419-434. https://doi.org/10.1007/978-3-642-14052-5_29

Matthieu Sozeau, Abhishek Anand, Simon Boulier, Cyril Cohen, Yannick Forster, Fabian Kunze, Gregory Malecha, Nicolas Tabareau, and Théo Winterhalter. 2019. The MetaCoq Project. (June 2019). https://hal.inria.fr/hal-02167423 working paper or preprint.

Philippe Suter, Ali Sinan Köksal, and Viktor Kuncak. 2011. Satisfiability Modulo Recursive Programs. In Static Analysis Symposium (SAS).

Nikhil Swamy, Catalin Hritcu, Chantal Keller, Aseem Rastogi, Antoine Delignat-Lavaud, Simon Forest, Karthikeyan Bhargavan, Cédric Fournet, Pierre-Yves Strub, Markulf Kohlweiss, Jean Karim Zinzindohoue, and Santiago Zanella Béguelin. 2016. Dependent types and multi-monadic effects in F. In Proceedings of the 43rd Annual ACM SIGPLAN-SIGACT Symposium on Principles of Programming Languages, POPL 2016, St. Petersburg, FL, USA, fanuary 20 - 22, 2016, Rastislav Bodík and Rupak Majumdar (Eds.). ACM, 256-270. https://doi.org/10.1145/2837614.2837655

Nikhil Swamy, Joel Weinberger, Cole Schlesinger, Juan Chen, and Benjamin Livshits. 2013. Verifying higher-order programs with the dijkstra monad. ACM SIGPLAN Conference on Programming Language Design and Implementation (2013), 387. https://doi.org/10.1145/2491956.2491978

W. W. Tait. 1967. Intensional interpretations of functionals of finite type I. Journal of Symbolic Logic 32, 2 (1967), $198-212$. https://doi.org/10.2307/2271658

Niki Vazou, Patrick Maxim Rondon, and Ranjit Jhala. 2013. Abstract Refinement Types. In European Symposium on Programming, ESOP. https://doi.org/10.1007/978-3-642-37036-6_13

Niki Vazou, Eric L. Seidel, Ranjit Jhala, Dimitrios Vytiniotis, and Simon Peyton-Jones. 2014. Refinement Types for Haskell. In International Conference on Functional Programming, ICFP. 269-282. https://doi.org/10.1145/2628136.2628161

Niki Vazou, Anish Tondwalkar, Vikraman Choudhury, Ryan G. Scott, Ryan R. Newton, Philip Wadler, and Ranjit Jhala. 2018. Refinement reflection: complete verification with SMT. PACMPL 2, POPL (2018), 53:1-53:31. https://doi.org/10.1145/ 3158141

Nicolas Voirol. 2019. Verifying Functional Programs. Ph.D. Dissertation. EPFL.

Nicolas Voirol, Etienne Kneuss, and Viktor Kuncak. 2015. Counter-example complete verification for higher-order functions. In Symposium on Scala, Scala 2015. 18-29. https://doi.org/10.1145/2774975.2774978

Hongwei Xi. 2001. Dependent Types for Program Termination Verification. In 16th Annual IEEE Symposium on Logic in Computer Science, Boston, Massachusetts, USA, June 16-19, 2001, Proceedings. 231-242. https://doi.org/10.1109/LICS.2001. 932500 